CRYSTALLOGRAPHIC COMMUNICATIONS

ISSN 2056-9890

Received 27 September 2015

Accepted 12 October 2015

Edited by M. Weil, Vienna University of Technology, Austria

Keywords: crystal structure; polyoxidomolybdate; Anderson-type anion; organic-inorganic hybrid

CCDC reference: 1430684 Supporting information: this article has supporting information at journals.iucr.org/e

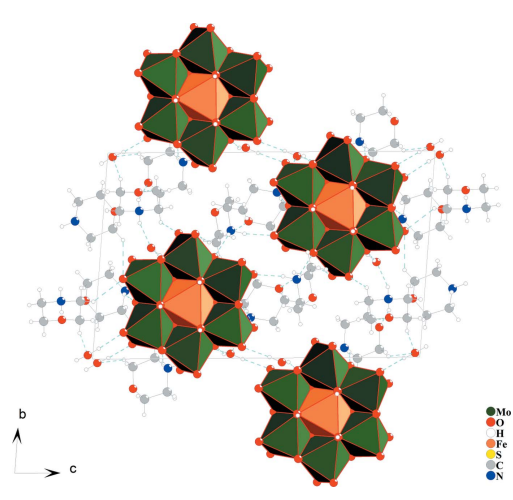

\section{Crystal structure of an organic-inorganic hybrid compound based on morpholinium cations and a $\beta$-type Anderson polyanion}

\author{
Tamara J. Lukianova,* Vasyl Kinzhybalo and Adam Pietraszko
}

Institute of Low Temperature and Structure Research, Polish Academy of Sciences, Okolna str. 2, PO Box 1410, 50-950 Wroclaw, Poland. *Correspondence e-mail: t.lukianova@int.pan.wroc.pl

A new organic-inorganic hybrid compound, pentamorpholinium hexahydrogen hexamolybdoferrate(III) sulfate 3.5-hydrate, $\left(\mathrm{C}_{4} \mathrm{H}_{10} \mathrm{NO}\right)_{5}\left[\mathrm{Fe}^{\mathrm{III}}(\mathrm{OH})_{6} \mathrm{Mo}_{6} \mathrm{O}_{18}\right]$ $\left(\mathrm{SO}_{4}\right) \cdot 3.5 \mathrm{H}_{2} \mathrm{O}$, was obtained from an aqueous solution. The polyoxidomolybdate (POM) anion is of the Anderson $\beta$-type with a central $\mathrm{Fe}^{\mathrm{III}}$ ion. Three of five crystallographically independent morpholinium cations are disordered over two sets of sites. An intricate network of intermolecular $\mathrm{N}-\mathrm{H} \cdots \mathrm{O}$ and $\mathrm{O}-\mathrm{H} \cdots \mathrm{O}$ interactions between cations, POMs, sulfate anions and non-coordinating water molecules creates a three-dimensional network structure.

\section{Chemical context}

Polyoxidometalates (POM) are attractive molecular building blocks used in the formation of multidimensional organicinorganic hybrid networks during self-organization processes (Pope \& Müller, 2001; Müller et al., 1998; Long et al., 2007). POMs play an important role in the design of new classes of functionalized materials not only because of their topological versatility and high dimensional architectures, but also due to their rich diversity of remarkable properties. Several related compounds with Anderson-type polyoxidometalate anions and organic cations, such as $\left(\mathrm{C}_{4} \mathrm{H}_{12} \mathrm{~N}_{2}\right)_{5}\left[\mathrm{Al}(\mathrm{OH})_{6} \mathrm{Mo}_{6} \mathrm{O}_{18}\right]_{2^{-}}$ $\left(\mathrm{SO}_{4}\right)_{2} \cdot 16 \mathrm{H}_{2} \mathrm{O}$ (Yang et al., 2009), $\left(\mathrm{C}_{4} \mathrm{H}_{10} \mathrm{NO}\right)_{3}\left[\mathrm{Cr}(\mathrm{OH})_{6^{-}}\right.$ $\left.\mathrm{Mo}_{6} \mathrm{O}_{18}\right] \cdot 4 \mathrm{H}_{2} \mathrm{O}$ (Yang et al., 2011), $\left(\mathrm{C}_{6} \mathrm{H}_{10} \mathrm{~N}_{3} \mathrm{O}_{2}\right)_{2} \mathrm{Na}\left(\mathrm{H}_{2} \mathrm{O}\right)_{2^{-}}$ $\left[\mathrm{Al}(\mathrm{OH})_{6} \mathrm{Mo}_{6} \mathrm{O}_{18}\right] \cdot 6 \mathrm{H}_{2} \mathrm{O}$ (Thabet et al., 2012) and other compounds with an $\mathrm{Fe}^{\mathrm{III}}$ central ion (Marcoux et al., 2003; Allain et al., 2008; Dessapt et al., 2011) have been reported. In $\beta$-type Anderson polyoxidoanions, which are characterized by a planar arrangement of the metal atoms, each $\mathrm{Mo}^{\mathrm{VI}}$ atom has two terminal oxygen atoms, two bridging $\mathrm{O}$ atoms and two bridging $\mathrm{OH}$ functions which make it highly reactive and easily coordinated by varieties of transition metal atoms in the anion.

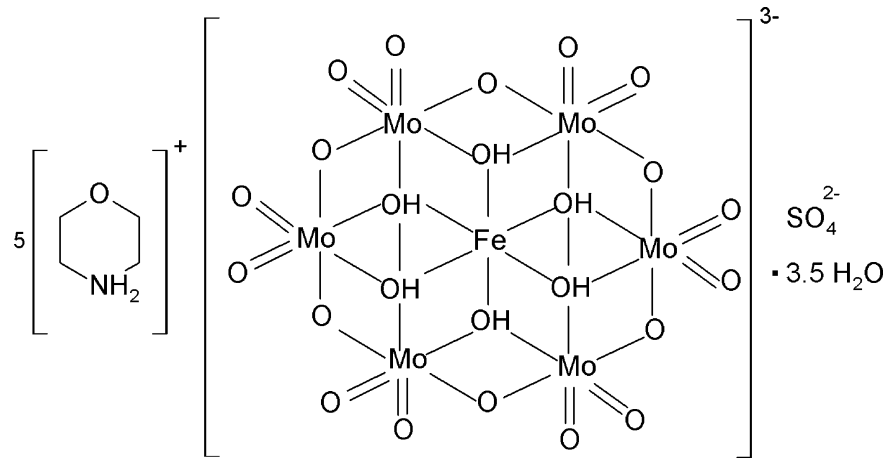




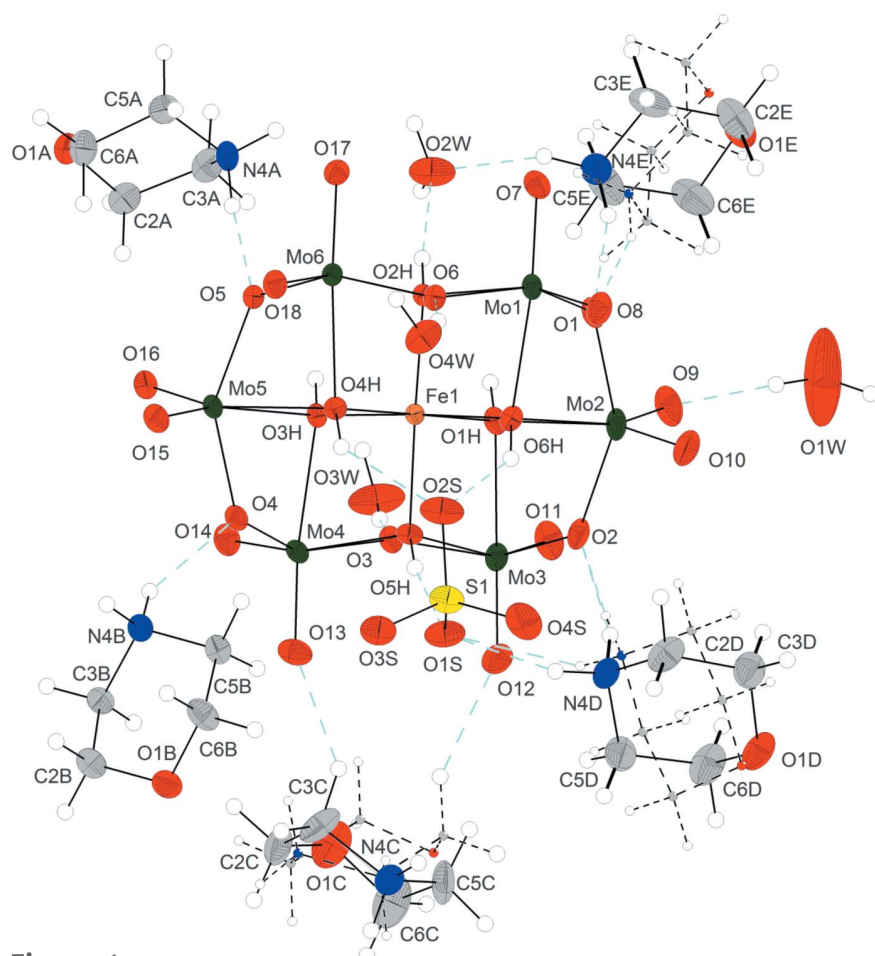

Figure 1

The molecular components in the structure of compound (I). Displacement ellipsoids are drawn at the $45 \%$ probability level. Hydrogen bonds are denoted by cyan dashed lines. Minor parts of the disordered cations are shown with dashed bonds.

Here we report synthesis and structure of the new organicinorganic hybrid compound $\left(\mathrm{C}_{4} \mathrm{H}_{10} \mathrm{NO}\right)_{5}\left[\mathrm{Fe}^{\mathrm{III}}(\mathrm{OH})_{6} \mathrm{Mo}_{6} \mathrm{O}_{18}\right]-$ $\left(\mathrm{SO}_{4}\right) \cdot 3.5 \mathrm{H}_{2} \mathrm{O},(\mathrm{I})$.

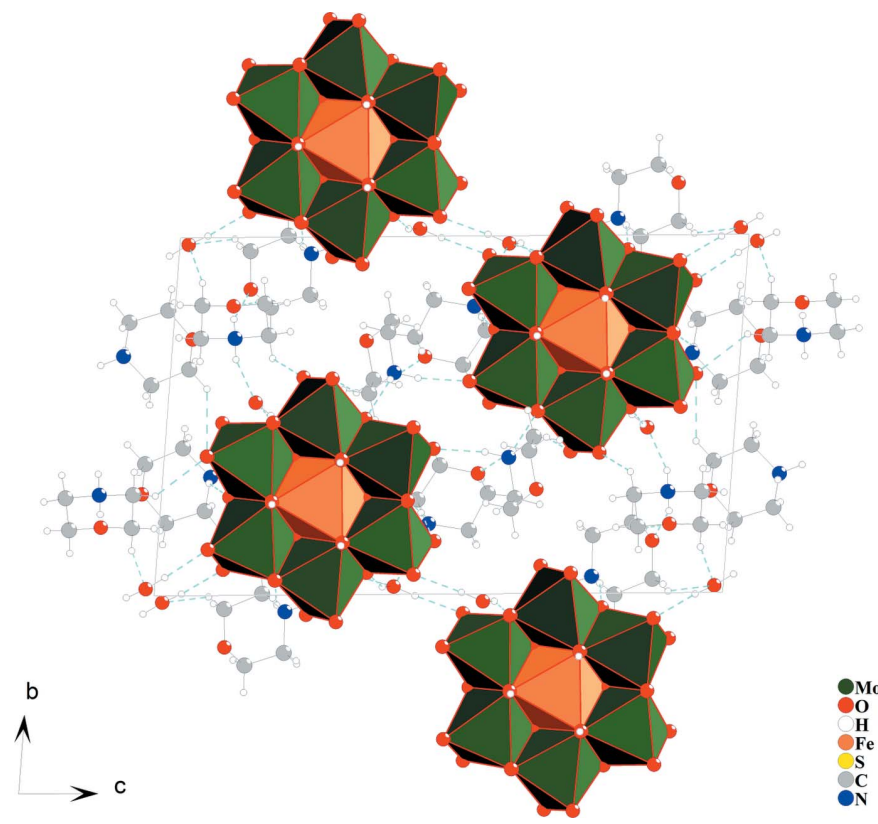

Figure 2

The crystal packing of compound (I) in a projection along [100], shown in the polyhedral mode for the POM anion. Orange and green octahedra are $\left[\mathrm{FeO}_{6}\right]$ and $\left[\mathrm{MoO}_{6}\right]$, respectively. Hydrogen bonds are shown as cyan dashed lines. Minor components of disorder for the morpholinium cations are omitted for clarity.
Table 1

Hydrogen-bond geometry $\left(\AA{ }^{\circ}\right)$.

\begin{tabular}{lllll}
\hline$D-\mathrm{H} \cdots A$ & $D-\mathrm{H}$ & $\mathrm{H} \cdots A$ & $D \cdots A$ & $D-\mathrm{H} \cdots A$ \\
\hline $\mathrm{O} 1 W-\mathrm{H} 1 W A \cdots \mathrm{O} 9$ & 0.84 & 1.94 & $2.781(11)$ & 178 \\
$\mathrm{O} 1 W-\mathrm{H} 1 W B \cdots \mathrm{O} 10^{\mathrm{i}}$ & 0.85 & 1.99 & $2.831(11)$ & 173 \\
$\mathrm{O} 2 W-\mathrm{H} 2 W A \cdots \mathrm{O} 4 W^{\mathrm{ii}}$ & 0.85 & 1.80 & $2.619(4)$ & 161 \\
$\mathrm{O} 2 W-\mathrm{H} 2 W B \cdots \mathrm{O} 2 S^{\mathrm{ii}}$ & 0.85 & 2.05 & $2.876(4)$ & 164 \\
$\mathrm{O} 2 W-\mathrm{H} 2 W B \cdots \mathrm{O} 4 S^{\mathrm{ii}}$ & 0.85 & 2.48 & $3.129(5)$ & 133 \\
$\mathrm{O} 3 W-\mathrm{H} 3 W A \cdots \mathrm{O} 1 S^{\mathrm{ii}}$ & 0.85 & 1.99 & $2.790(5)$ & 155 \\
$\mathrm{O} 3 W-\mathrm{H} 3 W B \cdots \mathrm{O} 3$ & 0.85 & 1.92 & $2.753(4)$ & 167 \\
$\mathrm{O} 4 W-\mathrm{H} 4 W A \cdots \mathrm{O} 17^{\mathrm{iii}}$ & 0.85 & 1.88 & $2.714(4)$ & 165 \\
$\mathrm{O} 4 W-\mathrm{H} 4 W B \cdots \mathrm{O} 6$ & 0.85 & 1.94 & $2.761(4)$ & 161 \\
$\mathrm{O} 1 H-\mathrm{H} 1 H \cdots \mathrm{O} 4 S^{\mathrm{ii}}$ & 1.00 & 1.69 & $2.673(4)$ & 165 \\
$\mathrm{O} 2 H-\mathrm{H} 2 H \cdots \mathrm{O} 2 W$ & 1.00 & 1.78 & $2.743(4)$ & 162 \\
$\mathrm{O} 3 H-\mathrm{H} 3 H \cdots \mathrm{O} 3 S^{\mathrm{ii}}$ & 1.00 & 1.61 & $2.602(4)$ & 174 \\
$\mathrm{O} 4 H-\mathrm{H} 4 H \cdots \mathrm{O} 2 S$ & 1.00 & 2.13 & $2.911(4)$ & 133 \\
$\mathrm{O} 5 H-\mathrm{H} 5 H \cdots \mathrm{O} 1 S$ & 1.00 & 1.69 & $2.672(4)$ & 165 \\
$\mathrm{O} 6 H-\mathrm{H} 6 H \cdots \mathrm{O} 2 S$ & 1.00 & 1.83 & $2.691(4)$ & 142 \\
$\mathrm{~N} 4 A-\mathrm{H} 4 A A \cdots \mathrm{O} 4 W^{\mathrm{ii}}$ & 0.91 & 2.34 & $3.102(5)$ & 141 \\
$\mathrm{~N} 4 A-\mathrm{H} 4 A B \cdots \mathrm{O} 5$ & 0.91 & 1.86 & $2.760(4)$ & 169 \\
$\mathrm{~N} 4 B-\mathrm{H} 4 B A \cdots \mathrm{O} 4$ & 0.91 & 2.00 & $2.761(4)$ & 140 \\
$\mathrm{~N} 4 B-\mathrm{H} 4 B A \cdots \mathrm{O} 1 A^{\mathrm{iv}}$ & 0.91 & 2.26 & $2.840(4)$ & 121 \\
$\mathrm{~N} 4 B-\mathrm{H} 4 B B \cdots \mathrm{O} 15^{\mathrm{v}}$ & 0.91 & 1.97 & $2.869(4)$ & 171 \\
$\mathrm{~N} 4 C-\mathrm{H} 4 C A \cdots \mathrm{O} 1 E^{\mathrm{vi}}$ & 0.91 & 1.97 & $2.817(7)$ & 155 \\
$\mathrm{~N} 41 C-\mathrm{H} 41 A \cdots \mathrm{O} 11 E^{\mathrm{vi}}$ & 0.91 & 1.92 & $2.54(5)$ & 124 \\
$\mathrm{~N} 4 E-\mathrm{H} 4 E A \cdots \mathrm{O} 1$ & 0.91 & 1.91 & $2.780(6)$ & 160 \\
$\mathrm{~N} 41 E-\mathrm{H} 41 D \cdots \mathrm{O} 1$ & 0.91 & 1.52 & $2.35(3)$ & 150 \\
$\mathrm{~N} 4 D-\mathrm{H} 4 D A \cdots \mathrm{O} 1 S$ & 0.91 & 1.99 & $2.866(8)$ & 162 \\
$\mathrm{~N} 4 D-\mathrm{H} 4 D B \cdots \mathrm{O} 2$ & 0.91 & 2.00 & $2.846(9)$ & 155 \\
$\mathrm{~N} 41 D-\mathrm{H} 41 E \cdots \mathrm{O} 2$ & 0.91 & 1.62 & $2.520(19)$ & 169 \\
\hline & & & & \\
\hline & & &
\end{tabular}

Symmetry codes: (i) $-x,-y+2,-z+2$; (ii) $x+1, y, z$; (iii) $-x,-y+2,-z+1$; (iv) $-x+1,-y+1,-z+1 ;$ (v) $-x,-y+1,-z+1$; (vi) $x-1, y-1, z$.

\section{Structural commentary}

The asymmetric unit of compound (I) is made up of one Anderson $\beta$-type polyoxidoanion, $\left[\mathrm{Fe}(\mathrm{OH})_{6} \mathrm{Mo}_{6} \mathrm{O}_{18}\right]^{3-}$, abbreviated in the following as $\left\{\mathrm{FeMo}_{6}\right\}$, five morpholinium cations $\left(\mathrm{C}_{4} \mathrm{H}_{10} \mathrm{NO}\right)^{+}$, one sulfate anion and four non-coordinating water molecules (Fig. 1). Three of the morpholinium cations are disordered over two sets of sites and one water molecule $(\mathrm{O} 1 W)$ shows half-occupancy. The $\left\{\mathrm{FeMo}_{6}\right\}$ anion is formed by six edge-sharing $\left[\mathrm{MoO}_{6}\right]$ octahedra, which are arranged hexagonally around the central $\left[\mathrm{Fe}(\mathrm{OH})_{6}\right]$ octahedron with bond lengths and angles that are within the expected ranges for this type of POM anion (Cao et al., 2007). The six hydroxyl groups of the Anderson-type polyoxoanion are involved as donor groups in hydrogen-bond formation with $\mathrm{O}$ atoms of the sulfate anions and the non-coordinating water molecules.

\section{Supramolecular features}

In the crystal structure of compound (I), hydrogen-bonding interactions between morpholinium cations, polyoxidoanions, sulfate anions and non-coordinating water molecules are of the types $\mathrm{O}-\mathrm{H} \cdots \mathrm{O}$ and $\mathrm{N}-\mathrm{H} \cdots \mathrm{O}$ (Table 1 ) and connect the discrete units into a three-dimensional supramolecular network. Hydrogen bonding is the dominating intermolecular interaction involved in the construction of this architecture and gives sufficient stabilization of its crystal structure. Figs. 2 


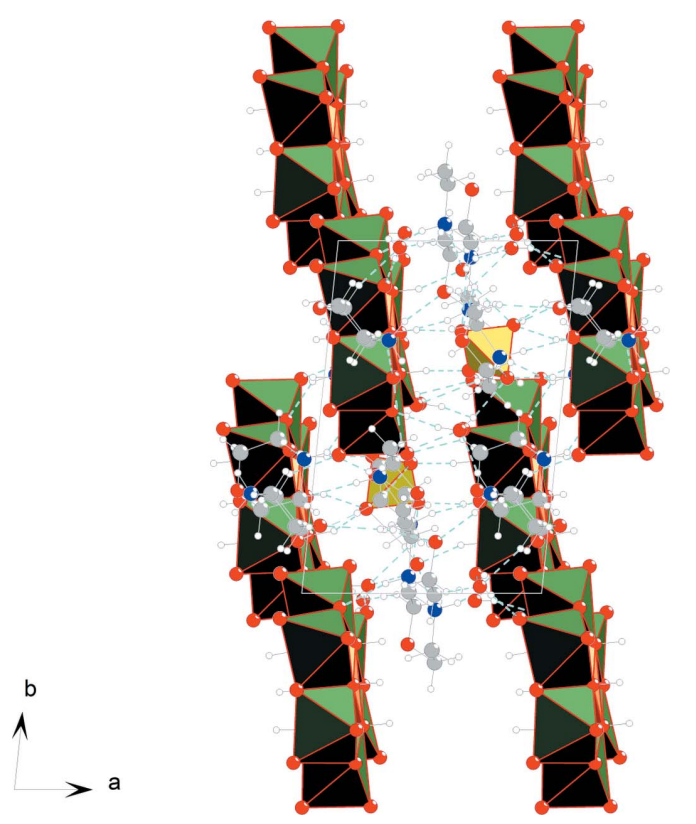

Figure 3

The crystal packing of compound (I) in a projection along [001].

and 3 shows the crystal packing with hydrogen-bonding interactions.

\section{Synthesis and crystallization}

The title compound was synthesized by the following procedure: $0.320 \mathrm{~g}(0.8 \mathrm{mmol})$ of iron(III) sulfate was dissolved in $10 \mathrm{ml}$ of double-distilled water. To this solution $4 \mathrm{ml}(5 \mathrm{mmol})$ of morpholine and $0.309 \mathrm{~g}(1.5 \mathrm{mmol})$ of $\mathrm{Na}_{2} \mathrm{MoO}_{4}$ were added during constant stirring. By the addition of $30 \% \mathrm{wt}$ sulfuric acid, the $\mathrm{pH}$ was adjusted to 2.5 . The resultant solution was filtered and the filtrate kept at room temperature. After few weeks, light-brown crystals were obtained.

\section{Refinement}

Crystal data, data collection and structure refinement details are summarized in Table 2. Three of the five crystallographically independent morpholinium cations are disordered, for which all atoms are distributed between two positions. The refined site occupation factor ratios are as follows: 0.857 (6):0.143 (6), 0.703 (9):0.297 (9) and 0.857 (6): 0.143 (6) for $\mathrm{O} 1 C-\mathrm{C} 6 C / \mathrm{O} 11 C-\mathrm{C} 61 C, \mathrm{O} 1 D-\mathrm{C} 6 D / \mathrm{O} 11 D-\mathrm{C} 61 D$ and $\mathrm{O} 1 E-\mathrm{C} 6 E / \mathrm{O} 11 E-\mathrm{C} 61 E$, respectively. All non-hydrogen atoms were refined anisotropically, except for the minor parts of the disordered morpholinium cations. The positions of the $\mathrm{H}$ atoms were initially located in difference Fourier maps. All $\mathrm{H}$ atoms were fixed at ideal positions, with $U_{\text {iso }}(\mathrm{H})=1.2 U_{\text {eq }}$ of the parent atom $\left(1.5 U_{\text {eq }}\right.$ for water $\mathrm{H}$ atoms). In the final refinement cycles, $\mathrm{H}$ atoms of the $\mathrm{O} 1 \mathrm{~W}$ water molecule were allowed to ride on the parent $\mathrm{O}$ atom (AFIX 3 in SHELXL2014; Sheldrick, 2015), H atoms of the other water molecules were fixed with the AFIX 6 instruction. For the
Table 2

Experimental details.

\begin{tabular}{|c|c|}
\hline \multicolumn{2}{|l|}{ Crystal data } \\
\hline Chemical formula & $\begin{array}{l}\left(\mathrm{C}_{4} \mathrm{H}_{10} \mathrm{NO}\right)_{5}\left[\mathrm{Fe}(\mathrm{OH})_{6} \mathrm{Mo}_{6} \mathrm{O}_{18}\right]- \\
\quad\left(\mathrm{SO}_{4}\right) \cdot 3.5 \mathrm{H}_{2} \mathrm{O}\end{array}$ \\
\hline$M_{\mathrm{r}}$ & 1621.30 \\
\hline Crystal system, space group & Triclinic, $P \overline{1}$ \\
\hline Temperature $(\mathrm{K})$ & 100 \\
\hline$a, b, c(\AA)$ & $8.900(3), 13.143(4), 20.778(6)$ \\
\hline$\alpha, \beta, \gamma\left({ }^{\circ}\right)$ & $84.92(3), 85.37(3), 83.70(3)$ \\
\hline$V\left(\AA^{3}\right)$ & $2400.1(13)$ \\
\hline$Z$ & 2 \\
\hline Radiation type & Мо $K \alpha$ \\
\hline$\mu\left(\mathrm{mm}^{-1}\right)$ & 1.97 \\
\hline Crystal size $(\mathrm{mm})$ & $0.27 \times 0.20 \times 0.12$ \\
\hline \multicolumn{2}{|l|}{ Data collection } \\
\hline Diffractometer & $\begin{array}{l}\text { Rigaku Oxford Diffraction } \\
\text { Xcalibur Atlas }\end{array}$ \\
\hline Absorption correction & $\begin{array}{l}\text { Analytical (CrysAlis PRO; Rigaku } \\
\text { Oxford Diffraction, 2015) }\end{array}$ \\
\hline$T_{\min }, T_{\max }$ & $0.708,0.819$ \\
\hline $\begin{array}{l}\text { No. of measured, independent and } \\
\text { observed }[I>2 \sigma(I)] \text { reflections }\end{array}$ & $37127,11708,9288$ \\
\hline$R_{\text {int }}$ & 0.038 \\
\hline$(\sin \theta / \lambda)_{\max }\left(\AA^{-1}\right)$ & 0.695 \\
\hline \multicolumn{2}{|l|}{ Refinement } \\
\hline$R\left[F^{2}>2 \sigma\left(F^{2}\right)\right], w R\left(F^{2}\right), S$ & $0.042,0.112,1.02$ \\
\hline No. of reflections & 11708 \\
\hline No. of parameters & 714 \\
\hline No. of restraints & 12 \\
\hline H-atom treatment & $\mathrm{H}$-atom parameters constrained \\
\hline$\Delta \rho_{\max }, \Delta \rho_{\min }\left(\mathrm{e} \AA^{-3}\right)$ & $1.98,-1.29$ \\
\hline
\end{tabular}

Computer programs: CrysAlis PRO (Rigaku Oxford Diffraction, 2015), SHELXS97 (Sheldrick, 2008), SHELXL2014 (Sheldrick, 2015), DIAMOND (Brandenburg, 1997) and $O L E X 2$ (Dolomanov et al., 2009).

minor component of disorder for morpholinium cation (O11C $>\mathrm{C} 61 C)$ the SAME instruction was used. Pairs of morpholinium cations (labelled $C$ and $E$ ) were initially refined with individual occupation factor variables which turned out to refine to the same value (taking into account standard uncertainties). As a result of the fact that disordered parts of these two cations are connected by hydrogen-bonding interactions, disorder was restrained with a common occupation factor variable in the final refinement. One of the $\mathrm{O}$ atom of a water molecule $(\mathrm{O} 1 W)$ is characterized by a significantly elongated displacement parameter. The occupation factor of this molecule was arbitrarily fixed at $50 \%$.

\section{Acknowledgements}

This research was supported by an ILT\&SR PAS grant for young scientists and PhD students funded by the Ministry of Science and Higher Education of Poland.

\section{References}

Allain, C., Favette, S., Chamoreau, L.-M., Vaissermann, J., Ruhlmann, L. \& Hasenknopf, B. (2008). Eur. J. Inorg. Chem. 2008, 34333441.

Brandenburg, K. (1997). DIAMOND. Crystal Impact GbR, Bonn, Germany. 
Cao, R. G., Liu, S. X., Xie, L. H., Pan, Y. B., Cao, J. F., Ren, Y. H. \& $\mathrm{Xu}, \mathrm{L}$. (2007). Inorg. Chem. 46, 3541-3547.

Dessapt, R., Gabard, M., Bujoli-Doeuff, M., Deniard, P. \& Jobic, S. (2011). Inorg. Chem. 50, 8790-8796.

Dolomanov, O. V., Bourhis, L. J., Gildea, R. J., Howard, J. A. K. \& Puschmann, H. (2009). J. Appl. Cryst. 42, 339-341.

Long, D.-L., Burkholder, E. \& Cronin, L. (2007). Chem. Soc. Rev. 36, 105-121.

Marcoux, P. R., Hasenknopf, B., Vaissermann, J. \& Gouzerh, P. (2003). Eur. J. Inorg. Chem. pp. 2406-2412.

Müller, A., Peters, F., Pope, M. T. \& Gatteschi, D. (1998). Chem. Rev. 98, 239-272.
Pope, M. T. \& Müller, A. (2001). In Polyoxometalate Chemistry: from Topology via Self-assembly to Applications. Dordrecht: Kluwer Academic Publishers.

Rigaku Oxford Diffraction (2015). CrysAlis PRO. Rigaku Oxford Diffraction, Yarnton, England.

Sheldrick, G. M. (2008). Acta Cryst. A64, 112-122.

Sheldrick, G. M. (2015). Acta Cryst. C71, 3-8.

Thabet, S., Ayed, B. \& Haddad, A. (2012). Mater. Res. Bull. 47, 37913796.

Yang, X.-D., Chen, Y.-G., Mirzaei, M., Salimi, A. R. \& Yao, F. (2009). Inorg. Chem. Commun. 12, 195-197.

Yang, Y.-Y., Song, Y., Liu, L.-Y. \& Qu, X.-S. (2011). Acta Cryst. E67, m776. 


\section{supporting information}

Acta Cryst. (2015). E71, 1345-1348 [https://doi.org/10.1107/S2056989015019246]

\section{Crystal structure of an organic-inorganic hybrid compound based on morpholinium cations and a $\beta$-type Anderson polyanion}

\section{Tamara J. Lukianova, Vasyl Kinzhybalo and Adam Pietraszko}

Computing details

Data collection: CrysAlis PRO (Rigaku Oxford Diffraction, 2015); cell refinement: CrysAlis PRO (Rigaku Oxford Diffraction, 2015); data reduction: CrysAlis PRO (Rigaku Oxford Diffraction, 2015); program(s) used to solve structure: SHELXS97 (Sheldrick, 2008); program(s) used to refine structure: SHELXL2014 (Sheldrick, 2015); molecular graphics: DIAMOND (Brandenburg, 1997); software used to prepare material for publication: OLEX2 (Dolomanov et al., 2009).

Pentamorpholinium hexahydrogen hexamolybdoferrate(III) sulfate 3.5-hydrate

\section{Crystal data}

$\left(\mathrm{C}_{4} \mathrm{H}_{10} \mathrm{NO}\right)_{5}\left[\mathrm{Fe}(\mathrm{OH})_{6} \mathrm{Mo}_{6} \mathrm{O}_{18}\right]\left(\mathrm{SO}_{4}\right) \cdot 3.5 \mathrm{H}_{2} \mathrm{O}$

$M_{r}=1621.30$

Triclinic, $P \overline{1}$

$a=8.900(3) \AA$

$b=13.143(4) \AA$

$c=20.778(6) \AA$

$\alpha=84.92(3)^{\circ}$

$\beta=85.37(3)^{\circ}$

$\gamma=83.70(3)^{\circ}$

$V=2400.1(13) \AA^{3}$

\section{Data collection}

Rigaku Oxford Diffraction Xcalibur Atlas diffractometer

Radiation source: fine-focus sealed X-ray tube, Enhance (Mo) X-ray Source

Graphite monochromator

Detector resolution: 10.6249 pixels $\mathrm{mm}^{-1}$

$\omega$ scans

Absorption correction: analytical

(CrysAlisPro; Rigaku Oxford Diffraction, 2015)

Refinement

Refinement on $F^{2}$

Least-squares matrix: full

$R\left[F^{2}>2 \sigma\left(F^{2}\right)\right]=0.042$

$w R\left(F^{2}\right)=0.112$

$S=1.02$

11708 reflections

714 parameters
$Z=2$

$F(000)=1608$

$D_{\mathrm{x}}=2.243 \mathrm{Mg} \mathrm{m}^{-3}$

Mo $K \alpha$ radiation, $\lambda=0.71073 \AA$

Cell parameters from 15587 reflections

$\theta=2.4-29.3^{\circ}$

$\mu=1.97 \mathrm{~mm}^{-1}$

$T=100 \mathrm{~K}$

Block, light brown

$0.27 \times 0.20 \times 0.12 \mathrm{~mm}$

$T_{\min }=0.708, T_{\max }=0.819$

37127 measured reflections

11708 independent reflections

9288 reflections with $I>2 \sigma(I)$

$R_{\text {int }}=0.038$

$\theta_{\max }=29.6^{\circ}, \theta_{\min }=2.4^{\circ}$

$h=-12 \rightarrow 11$

$k=-18 \rightarrow 17$

$l=-28 \rightarrow 28$

12 restraints

Primary atom site location: structure-invariant direct methods

Hydrogen site location: mixed

$\mathrm{H}$-atom parameters constrained

$w=1 /\left[\sigma^{2}\left(F_{\mathrm{o}}^{2}\right)+(0.0601 P)^{2}+2.7906 P\right]$

where $P=\left(F_{\mathrm{o}}^{2}+2 F_{\mathrm{c}}{ }^{2}\right) / 3$ 
$(\Delta / \sigma)_{\max }=0.001$

$\Delta \rho_{\max }=1.98$ e $\AA^{-3}$

$$
\Delta \rho_{\min }=-1.29 \text { e } \AA^{-3}
$$

Special details

Geometry. All e.s.d.'s (except the e.s.d. in the dihedral angle between two 1.s. planes) are estimated using the full covariance matrix. The cell e.s.d.'s are taken into account individually in the estimation of e.s.d.'s in distances, angles and torsion angles; correlations between e.s.d.'s in cell parameters are only used when they are defined by crystal symmetry. An approximate (isotropic) treatment of cell e.s.d.'s is used for estimating e.s.d.'s involving l.s. planes.

Fractional atomic coordinates and isotropic or equivalent isotropic displacement parameters $\left(\AA^{2}\right)$

\begin{tabular}{|c|c|c|c|c|c|}
\hline & $x$ & $y$ & $z$ & $U_{\text {iso }} * / U_{\text {eq }}$ & Occ. $(<1)$ \\
\hline Mo1 & $0.07030(4)$ & $0.98765(2)$ & $0.70745(2)$ & $0.01685(8)$ & \\
\hline Mo2 & $0.08559(5)$ & 0.86149 & $0.85537(2)$ & $0.02715(10)$ & \\
\hline Mo3 & $0.16217(4)$ & $0.60317(3)$ & $0.86796(2)$ & $0.02082(9)$ & \\
\hline Mo4 & $0.22287(4)$ & $0.47443(2)$ & $0.73545(2)$ & $0.01670(8)$ & \\
\hline Mo5 & $0.23106(4)$ & $0.60189(2)$ & $0.58822(2)$ & $0.01558(8)$ & \\
\hline Mo6 & $0.15245(3)$ & $0.85979(2)$ & $0.57545(2)$ & $0.01468(8)$ & \\
\hline O1 & $0.1659(3)$ & 0.9593 (2) & $0.78943(12)$ & $0.0246(6)$ & \\
\hline $\mathrm{O} 2$ & $0.0207(3)$ & $0.7260(2)$ & $0.87992(13)$ & $0.0268(7)$ & \\
\hline $\mathrm{O} 3$ & $0.2976(3)$ & $0.5184(2)$ & $0.81276(12)$ & $0.0185(5)$ & \\
\hline $\mathrm{O} 4$ & 0.1380 & $0.5093(2)$ & $0.65144(12)$ & $0.0192(6)$ & \\
\hline O5 & $0.2965(3)$ & $0.7388(2)$ & $0.56585(12)$ & $0.0180(5)$ & \\
\hline O6 & 0.0066 & $0.9393(2)$ & $0.62919(12)$ & $0.0169(5)$ & \\
\hline O7 & $0.1890(3)$ & $1.0768(2)$ & $0.67652(13)$ & $0.0245(6)$ & \\
\hline O8 & -0.0969 (3) & $1.0558(2)$ & $0.73014(13)$ & $0.0274(7)$ & \\
\hline O9 & $0.2118(5)$ & $0.8721(3)$ & $0.91123(14)$ & $0.0443(9)$ & \\
\hline $\mathrm{O} 1 \mathrm{~W}$ & $0.2590(12)$ & $0.9809(15)$ & $1.0154(8)$ & $0.184(9)$ & 0.5 \\
\hline H1WA & 0.2462 & 0.9492 & 0.9832 & $0.276^{*}$ & 0.5 \\
\hline H1WB & 0.2094 & 1.0047 & 1.0482 & $0.276^{*}$ & 0.5 \\
\hline $\mathrm{O} 10$ & $-0.0799(4)$ & 0.9283 & $0.88176(15)$ & $0.0438(9)$ & \\
\hline $\mathrm{O} 11$ & $0.2876(4)$ & $0.6120(2)$ & $0.92487(14)$ & $0.0346(8)$ & \\
\hline $\mathrm{O} 12$ & $0.0389(4)$ & $0.5191(2)$ & $0.90187(14)$ & $0.0314(7)$ & \\
\hline $\mathrm{O} 13$ & $0.0944(3)$ & $0.3921(2)$ & $0.76519(14)$ & $0.0299(7)$ & \\
\hline O14 & $0.3796(3)$ & $0.3960(2)$ & $0.71415(14)$ & $0.0274(6)$ & \\
\hline $\mathrm{O} 15$ & $0.1131(3)$ & $0.5942(2)$ & $0.52742(13)$ & $0.0230(6)$ & \\
\hline O16 & $0.3973(3)$ & $0.5344(2)$ & $0.56528(13)$ & $0.0226(6)$ & \\
\hline $\mathrm{O} 17$ & $0.2801(3)$ & $0.9456(2)$ & $0.54611(12)$ & $0.0206(6)$ & \\
\hline $\mathrm{O} 18$ & 0.0397 (3) & $0.8525(2)$ & $0.51406(12)$ & $0.0215(6)$ & \\
\hline $\mathrm{Fe} 1$ & $0.16272(5)$ & $0.73185(4)$ & $0.72223(2)$ & $0.01210(11)$ & \\
\hline $\mathrm{O} 1 \mathrm{H}$ & $0.2536(3)$ & $0.7399(2)$ & $0.80532(12)$ & $0.0185(6)$ & \\
\hline $\mathrm{H} 1 \mathrm{H}$ & 0.3645 & 0.7472 & 0.8042 & $0.022 *$ & \\
\hline $\mathrm{O} 2 \mathrm{H}$ & $0.2464(3)$ & $0.85564(19)$ & $0.67550(11)$ & $0.0147(5)$ & \\
\hline $\mathrm{H} 2 \mathrm{H}$ & 0.3564 & 0.8633 & 0.6777 & $0.018 *$ & \\
\hline $\mathrm{O} 3 \mathrm{H}$ & 0.3128 & 0.62367 (19) & $0.68738(12)$ & $0.0147(5)$ & \\
\hline $\mathrm{H} 3 \mathrm{H}$ & 0.4227 & 0.6314 & 0.6898 & $0.018^{*}$ & \\
\hline $\mathrm{O} 4 \mathrm{H}$ & 0.0625 & $0.7268(2)$ & $0.63805(11)$ & $0.0151(5)$ & \\
\hline $\mathrm{H} 4 \mathrm{H}$ & -0.0486 & 0.7198 & 0.6402 & $0.018^{*}$ & \\
\hline $\mathrm{O} 5 \mathrm{H}$ & 0.0654 & $0.6135(2)$ & $0.76894(12)$ & $0.0161(5)$ & \\
\hline
\end{tabular}




\begin{tabular}{|c|c|c|c|c|c|}
\hline $\mathrm{H} 5 \mathrm{H}$ & -0.0464 & 0.6125 & 0.7673 & $0.019^{*}$ & \\
\hline $\mathrm{O} 6 \mathrm{H}$ & $0.0001(3)$ & $0.8368(2)$ & $0.75643(12)$ & $0.0166(5)$ & \\
\hline $\mathrm{H} 6 \mathrm{H}$ & -0.1079 & 0.8232 & 0.7553 & $0.020^{*}$ & \\
\hline $\mathrm{S} 1$ & $-0.33015(11)$ & $0.68657(8)$ & $0.74350(5)$ & 0.0269 (2) & \\
\hline O1S & $-0.2351(3)$ & $0.6113(2)$ & $0.78520(15)$ & $0.0332(7)$ & \\
\hline $\mathrm{O} 2 \mathrm{~S}$ & $-0.2325(3)$ & $0.7606(2)$ & $0.70866(15)$ & $0.0349(8)$ & \\
\hline $\mathrm{O} 3 \mathrm{~S}$ & $-0.3991(3)$ & $0.6318(2)$ & $0.69703(15)$ & $0.0312(7)$ & \\
\hline O4S & $-0.4464(3)$ & $0.7430(3)$ & $0.78508(17)$ & $0.0405(8)$ & \\
\hline $\mathrm{O} 1 \mathrm{~A}$ & $0.7444(3)$ & $0.6640(2)$ & $0.44577(13)$ & $0.0257(6)$ & \\
\hline $\mathrm{C} 2 \mathrm{~A}$ & $0.7298(5)$ & $0.6408(4)$ & $0.5141(2)$ & $0.0291(10)$ & \\
\hline $\mathrm{H} 2 \mathrm{AA}$ & 0.8212 & 0.5969 & 0.5278 & $0.035^{*}$ & \\
\hline $\mathrm{H} 2 \mathrm{AB}$ & 0.6410 & 0.6018 & 0.5255 & $0.035^{*}$ & \\
\hline $\mathrm{C} 3 \mathrm{~A}$ & $0.7109(5)$ & $0.7361(4)$ & $0.5492(2)$ & $0.0342(11)$ & \\
\hline H3AA & 0.8029 & 0.7726 & 0.5407 & $0.041 *$ & \\
\hline $\mathrm{H} 3 \mathrm{AB}$ & 0.6962 & 0.7182 & 0.5964 & $0.041^{*}$ & \\
\hline N4A & $0.5764(4)$ & $0.8037(3)$ & $0.52633(17)$ & $0.0291(8)$ & \\
\hline H4AA & 0.5711 & 0.8649 & 0.5442 & $0.035^{*}$ & \\
\hline $\mathrm{H} 4 \mathrm{AB}$ & 0.4907 & 0.7738 & 0.5401 & $0.035^{*}$ & \\
\hline C5A & $0.5837(5)$ & $0.8224(3)$ & $0.4542(2)$ & $0.0289(9)$ & \\
\hline H5AA & 0.6676 & 0.8642 & 0.4391 & $0.035^{*}$ & \\
\hline H5AB & 0.4877 & 0.8601 & 0.4404 & $0.035^{*}$ & \\
\hline C6A & $0.6097(5)$ & $0.7196(4)$ & $0.4258(2)$ & $0.0295(10)$ & \\
\hline H6AA & 0.5231 & 0.6796 & 0.4397 & $0.035^{*}$ & \\
\hline H6AB & 0.6156 & 0.7302 & 0.3779 & $0.035^{*}$ & \\
\hline O1B & $-0.3116(3)$ & $0.2911(2)$ & $0.65919(15)$ & $0.0287(7)$ & \\
\hline $\mathrm{C} 2 \mathrm{~B}$ & $-0.2048(4)$ & $0.2376(3)$ & $0.6169(2)$ & $0.0257(9)$ & \\
\hline $\mathrm{H} 2 \mathrm{BA}$ & -0.2334 & 0.2535 & 0.5718 & $0.031^{*}$ & \\
\hline $\mathrm{H} 2 \mathrm{BB}$ & -0.2059 & 0.1627 & 0.6276 & $0.031 *$ & \\
\hline $\mathrm{C} 3 \mathrm{~B}$ & $-0.0465(4)$ & $0.2670(3)$ & $0.6222(2)$ & $0.0222(8)$ & \\
\hline H3BA & -0.0151 & 0.2484 & 0.6667 & $0.027^{*}$ & \\
\hline H3BB & 0.0263 & 0.2296 & 0.5918 & $0.027^{*}$ & \\
\hline N4B & $-0.0472(4)$ & $0.3801(2)$ & $0.60629(15)$ & $0.0206(7)$ & \\
\hline H4BA & 0.0454 & 0.3994 & 0.6124 & $0.025^{*}$ & \\
\hline H4BB & -0.0653 & 0.3957 & 0.5639 & $0.025 *$ & \\
\hline C5B & $-0.1655(4)$ & $0.4378(3)$ & $0.6478(2)$ & $0.0246(9)$ & \\
\hline H5BA & -0.1708 & 0.5121 & 0.6340 & $0.030 *$ & \\
\hline H5BB & -0.1395 & 0.4280 & 0.6935 & $0.030^{*}$ & \\
\hline C6B & $-0.3155(5)$ & $0.3992(3)$ & 0.6417 (2) & $0.0311(10)$ & \\
\hline H6BA & -0.3938 & 0.4361 & 0.6701 & $0.037 *$ & \\
\hline H6BB & -0.3439 & 0.4138 & 0.5965 & $0.037^{*}$ & \\
\hline $\mathrm{O} 1 \mathrm{C}$ & $0.0475(5)$ & $0.1916(5)$ & $0.8976(2)$ & $0.0507(14)$ & 0.857 (6) \\
\hline $\mathrm{C} 2 \mathrm{C}$ & $-0.0299(8)$ & $0.1850(5)$ & $0.8422(3)$ & $0.0418(16)$ & $0.857(6)$ \\
\hline $\mathrm{H} 2 \mathrm{CA}$ & -0.0784 & 0.1203 & 0.8465 & $0.050 *$ & $0.857(6)$ \\
\hline $\mathrm{H} 2 \mathrm{CB}$ & 0.0437 & 0.1832 & 0.8038 & $0.050^{*}$ & $0.857(6)$ \\
\hline $\mathrm{C} 3 \mathrm{C}$ & $-0.1492(6)$ & $0.2742(7)$ & 0.8318 & $0.0405(16)$ & $0.857(6)$ \\
\hline $\mathrm{H} 3 \mathrm{CA}$ & -0.1004 & 0.3386 & 0.8222 & $0.049 *$ & $0.857(6)$ \\
\hline $\mathrm{H} 3 \mathrm{CB}$ & -0.2065 & 0.2643 & 0.7943 & $0.049^{*}$ & $0.857(6)$ \\
\hline N4C & $-0.2543(6)$ & $0.2821(5)$ & 0.8907 (3) & $0.030(3)$ & $0.857(6)$ \\
\hline
\end{tabular}




\begin{tabular}{|c|c|c|c|c|c|}
\hline $\mathrm{H} 4 \mathrm{CA}$ & -0.3116 & 0.2284 & 0.8951 & $0.036^{*}$ & $0.857(6)$ \\
\hline $\mathrm{H} 4 \mathrm{CB}$ & -0.3178 & 0.3411 & 0.8864 & $0.036^{*}$ & 0.857 (6) \\
\hline C5C & $-0.1696(7)$ & $0.2820(6)$ & 0.9505 (3) & $0.0441(17)$ & 0.857 (6) \\
\hline H5CA & -0.1188 & 0.3455 & 0.9493 & $0.053^{*}$ & 0.857 (6) \\
\hline $\mathrm{H} 5 \mathrm{CB}$ & -0.2406 & 0.2788 & 0.9896 & $0.053^{*}$ & 0.857 (6) \\
\hline C6C & $-0.0539(8)$ & $0.1896(7)$ & 0.9524 (3) & $0.058(2)$ & 0.857 (6) \\
\hline H6CA & 0.0028 & 0.1877 & 0.9917 & $0.070 *$ & 0.857 (6) \\
\hline H6CB & -0.1061 & 0.1264 & 0.9551 & $0.070^{*}$ & 0.857 (6) \\
\hline $\mathrm{O} 11 \mathrm{C}$ & $-0.043(3)$ & $0.271(2)$ & $0.9549(11)$ & $0.057(8)^{*}$ & $0.143(6)$ \\
\hline $\mathrm{C} 21 \mathrm{C}$ & $0.063(3)$ & $0.234(3)$ & $0.9067(18)$ & $0.061(19)^{*}$ & $0.143(6)$ \\
\hline $\mathrm{H} 21 \mathrm{~A}$ & 0.1521 & 0.1960 & 0.9271 & $0.074 *$ & $0.143(6)$ \\
\hline $\mathrm{H} 21 \mathrm{~B}$ & 0.0988 & 0.2921 & 0.8782 & $0.074 *$ & $0.143(6)$ \\
\hline $\mathrm{C} 31 \mathrm{C}$ & $-0.007(5)$ & 0.163 & $0.867(2)$ & $0.10(3)^{*}$ & $0.143(6)$ \\
\hline $\mathrm{H} 31 \mathrm{~A}$ & 0.0682 & 0.1359 & 0.8336 & $0.122 *$ & $0.143(6)$ \\
\hline H31B & -0.0428 & 0.1046 & 0.8953 & $0.122 *$ & $0.143(6)$ \\
\hline N41C & $-0.137(4)$ & 0.223 & $0.8359(14)$ & $0.053(12)^{*}$ & $0.143(6)$ \\
\hline $\mathrm{H} 41 \mathrm{~A}$ & -0.1878 & 0.1801 & 0.8158 & $0.064^{*}$ & $0.143(6)$ \\
\hline H41B & -0.1014 & 0.2701 & 0.8053 & $0.064 *$ & $0.143(6)$ \\
\hline $\mathrm{C} 51 \mathrm{C}$ & $-0.245(3)$ & $0.278(4)$ & $0.884(2)$ & $0.07(4)^{*}$ & $0.143(6)$ \\
\hline H51A & -0.3003 & 0.2272 & 0.9119 & $0.087^{*}$ & $0.143(6)$ \\
\hline H51B & -0.3194 & 0.3259 & 0.8609 & $0.087^{*}$ & $0.143(6)$ \\
\hline C61C & $-0.158(3)$ & $0.3357(19)$ & $0.9249(12)$ & $0.020(6)^{*}$ & $0.143(6)$ \\
\hline H61A & -0.1118 & 0.3913 & 0.8975 & $0.024^{*}$ & $0.143(6)$ \\
\hline H61B & -0.2282 & 0.3678 & 0.9585 & $0.024^{*}$ & $0.143(6)$ \\
\hline O1E & $0.5358(5)$ & $1.1453(3)$ & $0.8694(2)$ & $0.0366(10)$ & $0.857(6)$ \\
\hline $\mathrm{C} 2 \mathrm{E}$ & $0.4163(7)$ & $1.1963(5)$ & 0.8317 (3) & $0.0443(16)$ & $0.857(6)$ \\
\hline H2EA & 0.4200 & 1.2716 & 0.8297 & $0.053 *$ & $0.857(6)$ \\
\hline H2EB & 0.3168 & 1.1807 & 0.8528 & $0.053^{*}$ & $0.857(6)$ \\
\hline $\mathrm{C} 3 \mathrm{E}$ & $0.4328(8)$ & $1.1618(4)$ & $0.7643(3)$ & $0.0389(15)$ & $0.857(6)$ \\
\hline H3EA & 0.5270 & 1.1840 & 0.7414 & $0.047 *$ & $0.857(6)$ \\
\hline H3EB & 0.3461 & 1.1935 & 0.7399 & $0.047^{*}$ & $0.857(6)$ \\
\hline N4E & $0.4379(5)$ & $1.0468(4)$ & $0.7675(2)$ & $0.0304(11)$ & $0.857(6)$ \\
\hline H4EA & 0.3454 & 1.0277 & 0.7825 & $0.037 *$ & $0.857(6)$ \\
\hline H4EB & 0.4586 & 1.0256 & 0.7268 & $0.037^{*}$ & $0.857(6)$ \\
\hline C5E & $0.5540(8)$ & $0.9958(5)$ & $0.8100(3)$ & $0.0457(17)$ & $0.857(6)$ \\
\hline H5EA & 0.6565 & 1.0085 & 0.7909 & $0.055^{*}$ & $0.857(6)$ \\
\hline H5EB & 0.5476 & 0.9208 & 0.8146 & $0.055^{*}$ & $0.857(6)$ \\
\hline C6E & $0.5269(9)$ & $1.0381(5)$ & $0.8748(3)$ & $0.0510(19)$ & $0.857(6)$ \\
\hline H6EA & 0.4254 & 1.0235 & 0.8941 & $0.061^{*}$ & $0.857(6)$ \\
\hline H6EB & 0.6033 & 1.0040 & 0.9039 & $0.061^{*}$ & $0.857(6)$ \\
\hline O11E & 0.610 & $1.148(2)$ & $0.8423(15)$ & $0.048(7)^{*}$ & $0.143(6)$ \\
\hline $\mathrm{C} 21 \mathrm{E}$ & $0.528(4)$ & $1.200(3)$ & $0.7879(18)$ & $0.044(9)^{*}$ & $0.143(6)$ \\
\hline $\mathrm{H} 21 \mathrm{C}$ & 0.5806 & 1.1787 & 0.7466 & $0.053^{*}$ & $0.143(6)$ \\
\hline $\mathrm{H} 21 \mathrm{D}$ & 0.5273 & 1.2749 & 0.7883 & $0.053^{*}$ & $0.143(6)$ \\
\hline $\mathrm{C} 31 \mathrm{E}$ & $0.363(4)$ & $1.170(3)$ & $0.7931(18)$ & $0.029(7)^{*}$ & $0.143(6)$ \\
\hline $\mathrm{H} 31 \mathrm{C}$ & 0.3099 & 1.1906 & 0.8344 & $0.035^{*}$ & $0.143(6)$ \\
\hline H31D & 0.3071 & 1.2063 & 0.7570 & $0.035^{*}$ & $0.143(6)$ \\
\hline N41E & $0.371(3)$ & 1.058 (2) & $0.7901(13)$ & $0.026(6)^{*}$ & $0.143(6)$ \\
\hline
\end{tabular}




\begin{tabular}{|c|c|c|c|c|c|}
\hline $\mathrm{H} 41 \mathrm{C}$ & 0.4108 & 1.0406 & 0.7504 & $0.032 *$ & $0.143(6)$ \\
\hline H41D & 0.2757 & 1.0376 & 0.7961 & $0.032 *$ & $0.143(6)$ \\
\hline C51E & $0.470(3)$ & $1.002(2)$ & $0.8430(13)$ & $0.016(6)^{*}$ & $0.143(6)$ \\
\hline $\mathrm{H} 51 \mathrm{C}$ & 0.4192 & 1.0122 & 0.8864 & $0.019 *$ & $0.143(6)$ \\
\hline H51D & 0.4835 & 0.9270 & 0.8376 & $0.019 *$ & $0.143(6)$ \\
\hline C61E & $0.617(4)$ & $1.041(3)$ & $0.8376(18)$ & $0.038(8)^{*}$ & $0.143(6)$ \\
\hline H61C & 0.6784 & 1.0058 & 0.8721 & $0.046^{*}$ & $0.143(6)$ \\
\hline H61D & 0.6700 & 1.0251 & 0.7954 & $0.046 *$ & $0.143(6)$ \\
\hline O1D & $-0.4396(8)$ & $0.7161(5)$ & $1.0345(3)$ & $0.0377(15)$ & $0.703(9)$ \\
\hline C2D & $-0.3989(9)$ & $0.7971(5)$ & 0.9881 & $0.039(2)$ & $0.703(9)$ \\
\hline H2DA & -0.3004 & 0.8185 & 0.9977 & $0.047^{*}$ & $0.703(9)$ \\
\hline H2DB & -0.4761 & 0.8570 & 0.9914 & $0.047 *$ & $0.703(9)$ \\
\hline C3D & $-0.3873(8)$ & $0.7643(6)$ & $0.9214(3)$ & $0.0361(19)$ & $0.703(9)$ \\
\hline H3DA & -0.3537 & 0.8205 & 0.8906 & $0.043 *$ & $0.703(9)$ \\
\hline H3DB & -0.4882 & 0.7495 & 0.9101 & $0.043 *$ & $0.703(9)$ \\
\hline N4D & $-0.2762(9)$ & $0.6697(6)$ & $0.9156(3)$ & $0.0303(15)$ & $0.703(9)$ \\
\hline H4DA & -0.2822 & 0.6448 & 0.8765 & $0.036^{*}$ & $0.703(9)$ \\
\hline H4DB & -0.1805 & 0.6869 & 0.9175 & $0.036 *$ & $0.703(9)$ \\
\hline C5D & $-0.3069(11)$ & $0.5889(6)$ & $0.9678(4)$ & $0.041(2)$ & $0.703(9)$ \\
\hline H5DA & -0.2219 & 0.5336 & 0.9674 & $0.049^{*}$ & $0.703(9)$ \\
\hline H5DB & -0.4002 & 0.5587 & 0.9600 & $0.049 *$ & $0.703(9)$ \\
\hline C6D & $-0.3255(10)$ & $0.6321(6)$ & $1.0326(3)$ & $0.046(2)$ & $0.703(9)$ \\
\hline H6DA & -0.3522 & 0.5776 & 1.0663 & $0.056^{*}$ & $0.703(9)$ \\
\hline H6DB & -0.2281 & 0.6549 & 1.0425 & $0.056^{*}$ & $0.703(9)$ \\
\hline O11D & $-0.4846(16)$ & $0.7037(12)$ & $1.0209(7)$ & $0.027(4)^{*}$ & $0.297(9)$ \\
\hline C21D & $-0.4828(19)$ & $0.7728(13)$ & $0.9674(8)$ & $0.032(4)^{*}$ & $0.297(9)$ \\
\hline $\mathrm{H} 21 \mathrm{E}$ & -0.5345 & 0.8400 & 0.9789 & $0.038 *$ & $0.297(9)$ \\
\hline $\mathrm{H} 21 \mathrm{~F}$ & -0.5382 & 0.7483 & 0.9333 & $0.038^{*}$ & $0.297(9)$ \\
\hline C31D & $-0.3180(18)$ & $0.7855(11)$ & $0.9415(7)$ & $0.025(3)^{*}$ & $0.297(9)$ \\
\hline H31E & -0.3177 & 0.8357 & 0.9030 & $0.030 *$ & $0.297(9)$ \\
\hline H31F & -0.2638 & 0.8122 & 0.9751 & $0.030 *$ & $0.297(9)$ \\
\hline N41D & $-0.241(2)$ & $0.6885(15)$ & $0.9241(9)$ & $0.027(5)^{*}$ & $0.297(9)$ \\
\hline $\mathrm{H} 41 \mathrm{E}$ & -0.1424 & 0.6955 & 0.9115 & $0.032 *$ & $0.297(9)$ \\
\hline $\mathrm{H} 41 \mathrm{~F}$ & -0.2853 & 0.6664 & 0.8906 & $0.032 *$ & $0.297(9)$ \\
\hline C51D & $-0.252(2)$ & $0.6104(15)$ & $0.9834(9)$ & $0.033(4)^{*}$ & $0.297(9)$ \\
\hline H51E & -0.2039 & 0.5422 & 0.9720 & $0.040 *$ & $0.297(9)$ \\
\hline H51F & -0.1978 & 0.6322 & 1.0188 & $0.040^{*}$ & $0.297(9)$ \\
\hline C61D & $-0.4176(16)$ & $0.6035(11)$ & $1.0057(7)$ & $0.025(4)^{*}$ & $0.297(9)$ \\
\hline H61E & -0.4711 & 0.5790 & 0.9710 & $0.030 *$ & $0.297(9)$ \\
\hline H61F & -0.4259 & 0.5543 & 1.0444 & $0.030 *$ & $0.297(9)$ \\
\hline $\mathrm{O} 2 \mathrm{~W}$ & 0.5313 & $0.9178(3)$ & $0.67269(17)$ & $0.0394(8)$ & \\
\hline H2WA & 0.5813 & 0.9500 & 0.6425 & $0.059 *$ & \\
\hline H2WB & 0.5885 & 0.8684 & 0.6897 & $0.059 *$ & \\
\hline $\mathrm{O} 3 \mathrm{~W}$ & $0.5901(4)$ & $0.4619(3)$ & $0.8460(2)$ & $0.0525(11)$ & \\
\hline H3WA & 0.6398 & 0.4979 & 0.8176 & $0.079^{*}$ & \\
\hline H3WB & 0.4967 & 0.4702 & 0.8386 & $0.079 *$ & \\
\hline O4W & -0.2744 & 0.9785 & $0.57958(14)$ & $0.0327(7)$ & \\
\hline H4WA & -0.2595 & 1.0042 & 0.5408 & $0.049 *$ & \\
\hline
\end{tabular}


Atomic displacement parameters $\left(\AA^{2}\right)$

\begin{tabular}{|c|c|c|c|c|c|c|}
\hline & $U^{11}$ & $U^{22}$ & $U^{33}$ & $U^{12}$ & $U^{13}$ & $U^{23}$ \\
\hline Mo1 & $0.02266(17)$ & $0.01483(17)$ & $0.01282(15)$ & $0.00194(12)$ & $-0.00431(12)$ & $-0.00177(12)$ \\
\hline Mo2 & $0.0502(2)$ & 0.01917 (19) & $0.01084(16)$ & $0.00373(16)$ & $-0.00339(15)$ & $-0.00225(14)$ \\
\hline Mo3 & 0.03058 (19) & $0.01924(18)$ & $0.01175(15)$ & $0.00277(14)$ & $-0.00552(13)$ & $0.00067(13)$ \\
\hline Mo4 & $0.02022(17)$ & $0.01442(17)$ & $0.01648(16)$ & $-0.00286(12)$ & $-0.00614(12)$ & $-0.00127(13)$ \\
\hline Mo5 & $0.01895(16)$ & $0.01605(17)$ & $0.01302(15)$ & $-0.00288(12)$ & $-0.00493(12)$ & $-0.00354(12)$ \\
\hline Mo6 & $0.01686(16)$ & $0.01677(17)$ & $0.01099(14)$ & $-0.00214(12)$ & $-0.00403(11)$ & $-0.00110(12)$ \\
\hline $\mathrm{O} 1$ & $0.0416(17)$ & $0.0191(15)$ & $0.0147(13)$ & $-0.0020(12)$ & $-0.0111(11)$ & $-0.0027(11)$ \\
\hline $\mathrm{O} 2$ & $0.0430(18)$ & $0.0202(15)$ & $0.0137(13)$ & $0.0069(13)$ & $0.0033(12)$ & $0.0001(11)$ \\
\hline $\mathrm{O} 3$ & $0.0209(13)$ & $0.0187(14)$ & $0.0166(12)$ & $0.0005(10)$ & $-0.0082(10)$ & $-0.0019(11)$ \\
\hline $\mathrm{O} 4$ & $0.0245(14)$ & $0.0193(14)$ & $0.0157(12)$ & $-0.0051(11)$ & $-0.0072(10)$ & $-0.0030(11)$ \\
\hline O5 & $0.0194(13)$ & $0.0176(14)$ & $0.0170(12)$ & $-0.0020(10)$ & $0.0006(10)$ & $-0.0037(11)$ \\
\hline O6 & $0.0175(13)$ & $0.0188(14)$ & $0.0140(12)$ & $0.0016(10)$ & $-0.0040(10)$ & $-0.0014(10)$ \\
\hline $\mathrm{O} 7$ & $0.0348(16)$ & $0.0180(15)$ & $0.0213(14)$ & $-0.0024(12)$ & $-0.0085(12)$ & $-0.0005(12)$ \\
\hline $\mathrm{O} 8$ & $0.0317(16)$ & $0.0245(16)$ & $0.0231(14)$ & $0.0089(12)$ & $0.0011(12)$ & $-0.0028(12)$ \\
\hline O9 & $0.088(3)$ & 0.0299 (19) & $0.0172(15)$ & $-0.0044(18)$ & $-0.0195(16)$ & $-0.0034(13)$ \\
\hline $\mathrm{O} 1 \mathrm{~W}$ & $0.047(6)$ & $0.34(2)$ & $0.204(15)$ & $-0.034(10)$ & $0.009(8)$ & $-0.222(16)$ \\
\hline $\mathrm{O} 10$ & $0.073(3)$ & $0.0252(18)$ & $0.0257(16)$ & $0.0091(16)$ & $0.0178(16)$ & $0.0002(14)$ \\
\hline O11 & $0.056(2)$ & $0.0290(17)$ & $0.0201(14)$ & $0.0045(15)$ & $-0.0213(14)$ & $-0.0012(13)$ \\
\hline $\mathrm{O} 12$ & $0.0392(18)$ & $0.0264(17)$ & $0.0249(15)$ & $0.0032(13)$ & $0.0026(13)$ & $0.0052(13)$ \\
\hline $\mathrm{O} 13$ & $0.0352(17)$ & $0.0300(17)$ & $0.0277(15)$ & $-0.0146(13)$ & $-0.0124(13)$ & $0.0038(13)$ \\
\hline $\mathrm{O} 14$ & $0.0336(16)$ & $0.0221(16)$ & $0.0258(15)$ & $0.0034(12)$ & $-0.0055(12)$ & $-0.0035(12)$ \\
\hline O15 & $0.0272(15)$ & $0.0242(15)$ & $0.0198(13)$ & $-0.0051(12)$ & $-0.0099(11)$ & $-0.0040(12)$ \\
\hline O16 & $0.0241(14)$ & $0.0223(15)$ & $0.0219(14)$ & $-0.0012(11)$ & $-0.0027(11)$ & $-0.0060(12)$ \\
\hline O17 & $0.0211(14)$ & $0.0205(15)$ & 0.0195 (13) & $-0.0003(11)$ & $-0.0007(10)$ & $-0.0001(11)$ \\
\hline O18 & $0.0253(15)$ & $0.0234(15)$ & $0.0163(13)$ & $-0.0007(11)$ & $-0.0062(11)$ & $-0.0028(11)$ \\
\hline $\mathrm{Fe} 1$ & $0.0122(2)$ & $0.0141(3)$ & $0.0106(2)$ & -0.00148 (19) & $-0.00381(18)$ & $-0.0014(2)$ \\
\hline $\mathrm{O} 1 \mathrm{H}$ & 0.0199 (13) & $0.0204(14)$ & $0.0167(12)$ & $-0.0009(11)$ & $-0.0091(10)$ & $-0.0042(11)$ \\
\hline $\mathrm{O} 2 \mathrm{H}$ & $0.0148(12)$ & $0.0162(13)$ & 0.0139 (12) & $-0.0024(10)$ & $-0.0046(9)$ & $-0.0015(10)$ \\
\hline $\mathrm{O} 3 \mathrm{H}$ & $0.0130(12)$ & $0.0162(13)$ & $0.0154(12)$ & $0.0002(9)$ & $-0.0038(9)$ & $-0.0041(10)$ \\
\hline $\mathrm{O} 4 \mathrm{H}$ & 0.0139 (12) & $0.0194(14)$ & $0.0129(12)$ & $-0.0034(10)$ & $-0.0058(9)$ & $-0.0005(10)$ \\
\hline $\mathrm{O} 5 \mathrm{H}$ & $0.0134(12)$ & $0.0171(14)$ & $0.0183(12)$ & $-0.0038(10)$ & $-0.0063(10)$ & $0.0027(11)$ \\
\hline $\mathrm{O} 6 \mathrm{H}$ & $0.0148(12)$ & $0.0197(14)$ & $0.0145(12)$ & $0.0024(10)$ & $-0.0025(9)$ & $-0.0003(11)$ \\
\hline $\mathrm{S} 1$ & $0.0144(5)$ & 0.0299 (6) & $0.0369(6)$ & -0.0038 & $-0.0080(4)$ & $0.0019(5)$ \\
\hline O1S & $0.0202(15)$ & 0.0391 (19) & $0.0395(18)$ & $-0.0083(13)$ & $-0.0063(13)$ & $0.0112(15)$ \\
\hline $\mathrm{O} 2 \mathrm{~S}$ & $0.0244(15)$ & 0.0367 (19) & 0.0445 (19) & -0.0103 & $-0.0176(13)$ & $0.0150(15)$ \\
\hline $\mathrm{O} 3 \mathrm{~S}$ & $0.0184(14)$ & $0.0361(18)$ & $0.0401(18)$ & $-0.0065(12)$ & $-0.0052(12)$ & -0.0003 \\
\hline $\mathrm{O} 4 \mathrm{~S}$ & $0.0203(16)$ & $0.046(2)$ & $0.060(2)$ & $-0.0082(14)$ & $-0.0074(15)$ & $-0.0161(17)$ \\
\hline O1A & $0.0239(15)$ & $0.0290(16)$ & $0.0239(14)$ & $0.0007(12)$ & $-0.0022(11)$ & $-0.0054(12)$ \\
\hline $\mathrm{C} 2 \mathrm{~A}$ & $0.026(2)$ & $0.035(3)$ & $0.026(2)$ & $-0.0006(18)$ & $-0.0054(17)$ & 0.0016 (19) \\
\hline C3A & $0.029(2)$ & $0.052(3)$ & $0.025(2)$ & $-0.011(2)$ & $-0.0061(17)$ & $-0.007(2)$ \\
\hline N4A & 0.0265 (19) & $0.031(2)$ & $0.0321(19)$ & $-0.0077(15)$ & $0.0075(15)$ & $-0.0162(16)$ \\
\hline C5A & $0.026(2)$ & $0.025(2)$ & $0.034(2)$ & $0.0018(17)$ & $0.0024(17)$ & 0.0009 (19) \\
\hline C6A & $0.030(2)$ & $0.038(3)$ & $0.022(2)$ & 0.0001 (19) & $-0.0096(17)$ & $-0.0072(19)$ \\
\hline
\end{tabular}




$\begin{array}{lllllll}\text { O1B } & 0.0245(15) & 0.0247(16) & 0.0373(17) & -0.0046(12) & 0.0005(12) & -0.0047(13) \\ \text { C2B } & 0.023(2) & 0.023(2) & 0.031(2) & -0.0014(16) & -0.0095(17) & -0.0026(18) \\ \text { C3B } & 0.023(2) & 0.019(2) & 0.025(2) & -0.0010(15) & -0.0053(15) & -0.0004(16) \\ \text { N4B } & 0.0208(16) & 0.0244(18) & 0.0179(15) & -0.0031(13) & -0.0077(12) & -0.0024(14) \\ \text { C5B } & 0.026(2) & 0.022(2) & 0.026(2) & -0.0014(16) & -0.0028(16) & -0.0049(17) \\ \text { C6B } & 0.025(2) & 0.022(2) & 0.047(3) & -0.0005(17) & -0.0061(19) & -0.007(2) \\ \text { O1C } & 0.036(2) & 0.072(4) & 0.040(3) & 0.013(2) & -0.008(2) & -0.002(3) \\ \text { C2C } & 0.054(4) & 0.035(3) & 0.031(3) & 0.011(3) & 0.011(3) & -0.005(3) \\ \text { C3C } & 0.032(3) & 0.056(5) & 0.026(3) & 0.004(3) & 0.006(2) & 0.015(3) \\ \text { N4C } & 0.025(3) & 0.036(4) & 0.029(3) & -0.0039(18) & 0.0011(18) & -0.005(2) \\ \text { C5C } & 0.037(3) & 0.064(5) & 0.036(3) & -0.009(3) & 0.001(2) & -0.034(3) \\ \text { C6C } & 0.055(4) & 0.089(6) & 0.029(3) & 0.010(4) & -0.016(3) & -0.007(3) \\ \text { O1E } & 0.048(3) & 0.030(2) & 0.034(2) & -0.0058(18) & -0.019(2) & -0.0044(17) \\ \text { C2E } & 0.049(4) & 0.033(3) & 0.054(4) & -0.001(3) & -0.021(3) & -0.009(3) \\ \text { C3E } & 0.050(4) & 0.035(3) & 0.036(3) & -0.021(3) & -0.025(3) & 0.011(3) \\ \text { N4E } & 0.025(2) & 0.039(3) & 0.030(2) & -0.009(2) & -0.007(2) & -0.006(2) \\ \text { C5E } & 0.050(4) & 0.033(3) & 0.058(4) & 0.002(3) & -0.031(3) & -0.008(3) \\ \text { C6E } & 0.085(5) & 0.034(4) & 0.038(3) & -0.018(3) & -0.030(3) & 0.010(3) \\ \text { O1D } & 0.041(4) & 0.044(3) & 0.022(3) & 0.012(3) & 0.002(3) & 0.006(2) \\ \text { C2D } & 0.048(5) & 0.030(4) & 0.036(4) & 0.007(3) & 0.001(3) & -0.002(3) \\ \text { C3D } & 0.034(4) & 0.043(4) & 0.026(3) & 0.009(3) & -0.002(3) & 0.010(3) \\ \text { N4D } & 0.035(4) & 0.037(4) & 0.019(3) & -0.008(3) & 0.001(3) & -0.005(3) \\ \text { C5D } & 0.063(6) & 0.024(4) & 0.033(4) & 0.003(4) & 0.002(4) & 0.001(3) \\ \text { C6D } & 0.059(5) & 0.044(5) & 0.029(4) & 0.017(4) & -0.001(3) & 0.008(3) \\ \text { O2W } & 0.0255(16) & 0.044(2) & 0.049(2) & -0.0111(14) & -0.0154(14) & 0.0164(17) \\ \text { O3W } & 0.0206(16) & 0.064(3) & 0.068(3) & -0.0085(17) & -0.0126(16) & 0.032(2) \\ \text { O4W } & 0.0242(15) & 0.048(2) & 0.0244(15) & -0.0006(14) & -0.0078(12) & 0.0055(15) \\ & & & & & & \end{array}$

Geometric parameters $\left(\AA,{ }^{\circ}\right)$

\begin{tabular}{llll}
\hline Mo1-O1 & $1.954(3)$ & N4C-H4CA & 0.9100 \\
Mo1-O6 & $1.944(3)$ & N4C-H4CB & 0.9100 \\
Mo1-O7 & $1.712(3)$ & N4C-C5C & $1.503(8)$ \\
Mo1-O8 & $1.705(3)$ & C5C-H5CA & 0.9900 \\
Mo1-O2H & $2.312(3)$ & C5C-H5CB & 0.9900 \\
Mo1-O6H & $2.273(3)$ & C5C-C6C & $1.503(10)$ \\
Mo2-O1 & $1.942(3)$ & C6C-H6CA & 0.9900 \\
Mo2-O2 & $1.944(3)$ & C6C-H6CB & 0.9900 \\
Mo2-O9 & $1.703(3)$ & O11C-C21C & $1.404(14)$ \\
Mo2-O10 & $1.706(3)$ & O11C-C61C & $1.403(12)$ \\
Mo2-O1H & $2.322(3)$ & C21C-H21A & 0.9900 \\
Mo2-O6H & $2.309(3)$ & C21C-H21B & 0.9900 \\
Mo3-O2 & $1.957(3)$ & C21C-C31C & $1.511(13)$ \\
Mo3-O3 & $1.928(3)$ & C31C-H31A & 0.9900 \\
Mo3-O11 & $1.710(3)$ & C31C-H31B & 0.9900 \\
Mo3-O12 & $1.710(3)$ & C31C-N41C & $1.484(13)$ \\
Mo3-O1H & $2.307(3)$ & N41C-H41A & 0.9100 \\
Mo3-O5H & $2.281(3)$ & N41C-H41B & 0.9100
\end{tabular}




\begin{tabular}{|c|c|}
\hline Mo4-O3 & $1.944(3)$ \\
\hline Mo4-O4 & $1.955(3)$ \\
\hline Mo4-O13 & $1.703(3)$ \\
\hline Mo4-O14 & $1.694(3)$ \\
\hline $\mathrm{Mo} 4-\mathrm{O} 3 \mathrm{H}$ & $2.314(3)$ \\
\hline $\mathrm{Mo} 4-\mathrm{O} 5 \mathrm{H}$ & $2.302(3)$ \\
\hline Mo5-O4 & $1.912(3)$ \\
\hline Mo5-O5 & $1.957(3)$ \\
\hline Mo5-O15 & $1.723(3)$ \\
\hline Mo5-O16 & $1.696(3)$ \\
\hline $\mathrm{Mo} 5-\mathrm{O} 3 \mathrm{H}$ & $2.291(3)$ \\
\hline Mo5-O4H & $2.351(3)$ \\
\hline Mo6-O5 & $1.944(3)$ \\
\hline Mo6-O6 & $1.923(3)$ \\
\hline Mo6-O17 & $1.727(3)$ \\
\hline Mo6-O18 & $1.701(3)$ \\
\hline Mo6-O2H & $2.297(2)$ \\
\hline Mo6-O4H & $2.264(3)$ \\
\hline $\mathrm{O} 1 \mathrm{~W}-\mathrm{H} 1 \mathrm{WA}$ & 0.8397 \\
\hline $\mathrm{O} 1 \mathrm{~W}-\mathrm{H} 1 \mathrm{WB}$ & 0.8468 \\
\hline $\mathrm{Fe} 1-\mathrm{O} 1 \mathrm{H}$ & $1.981(3)$ \\
\hline $\mathrm{Fe} 1-\mathrm{O} 2 \mathrm{H}$ & $2.003(3)$ \\
\hline $\mathrm{Fe} 1-\mathrm{O} 3 \mathrm{H}$ & $1.985(3)$ \\
\hline $\mathrm{Fe} 1-\mathrm{O} 4 \mathrm{H}$ & $2.036(3)$ \\
\hline $\mathrm{Fe} 1-\mathrm{O} 5 \mathrm{H}$ & $2.001(3)$ \\
\hline $\mathrm{Fe} 1-\mathrm{O} 6 \mathrm{H}$ & $2.018(3)$ \\
\hline $\mathrm{O} 1 \mathrm{H}-\mathrm{H} 1 \mathrm{H}$ & 1.0000 \\
\hline $\mathrm{O} 2 \mathrm{H}-\mathrm{H} 2 \mathrm{H}$ & 1.0000 \\
\hline $\mathrm{O} 3 \mathrm{H}-\mathrm{H} 3 \mathrm{H}$ & 1.0000 \\
\hline $\mathrm{O} 4 \mathrm{H}-\mathrm{H} 4 \mathrm{H}$ & 1.0000 \\
\hline $\mathrm{O} 5 \mathrm{H}-\mathrm{H} 5 \mathrm{H}$ & 1.0000 \\
\hline $\mathrm{O} 6 \mathrm{H}-\mathrm{H} 6 \mathrm{H}$ & 1.0000 \\
\hline $\mathrm{S} 1-\mathrm{O} 1 \mathrm{~S}$ & $1.493(3)$ \\
\hline $\mathrm{S} 1-\mathrm{O} 2 \mathrm{~S}$ & $1.478(3)$ \\
\hline $\mathrm{S} 1-\mathrm{O} 3 \mathrm{~S}$ & $1.464(3)$ \\
\hline $\mathrm{S} 1-\mathrm{O} 4 \mathrm{~S}$ & $1.473(4)$ \\
\hline $\mathrm{O} 1 \mathrm{~A}-\mathrm{C} 2 \mathrm{~A}$ & $1.423(5)$ \\
\hline $\mathrm{O} 1 \mathrm{~A}-\mathrm{C} 6 \mathrm{~A}$ & $1.406(5)$ \\
\hline $\mathrm{C} 2 \mathrm{~A}-\mathrm{H} 2 \mathrm{AA}$ & 0.9900 \\
\hline $\mathrm{C} 2 \mathrm{~A}-\mathrm{H} 2 \mathrm{AB}$ & 0.9900 \\
\hline $\mathrm{C} 2 \mathrm{~A}-\mathrm{C} 3 \mathrm{~A}$ & $1.492(7)$ \\
\hline $\mathrm{C} 3 \mathrm{~A}-\mathrm{H} 3 \mathrm{AA}$ & 0.9900 \\
\hline $\mathrm{C} 3 \mathrm{~A}-\mathrm{H} 3 \mathrm{AB}$ & 0.9900 \\
\hline $\mathrm{C} 3 \mathrm{~A}-\mathrm{N} 4 \mathrm{~A}$ & $1.494(6)$ \\
\hline $\mathrm{N} 4 \mathrm{~A}-\mathrm{H} 4 \mathrm{AA}$ & 0.9100 \\
\hline $\mathrm{N} 4 \mathrm{~A}-\mathrm{H} 4 \mathrm{AB}$ & 0.9100 \\
\hline $\mathrm{N} 4 \mathrm{~A}-\mathrm{C} 5 \mathrm{~A}$ & $1.494(5)$ \\
\hline $\mathrm{C} 5 \mathrm{~A}-\mathrm{H} 5 \mathrm{AA}$ & 0.9900 \\
\hline
\end{tabular}

\begin{tabular}{|c|c|}
\hline $\mathrm{N} 41 \mathrm{C}-\mathrm{C} 51 \mathrm{C}$ & $1.500(13)$ \\
\hline C51C-H51A & 0.9900 \\
\hline $\mathrm{C} 51 \mathrm{C}-\mathrm{H} 51 \mathrm{~B}$ & 0.9900 \\
\hline $\mathrm{C} 51 \mathrm{C}-\mathrm{C} 61 \mathrm{C}$ & $1.501(14)$ \\
\hline C61C-H61A & 0.9900 \\
\hline C61C-H61B & 0.9900 \\
\hline $\mathrm{O} 1 \mathrm{E}-\mathrm{C} 2 \mathrm{E}$ & $1.442(7)$ \\
\hline $\mathrm{O} 1 \mathrm{E}-\mathrm{C} 6 \mathrm{E}$ & $1.413(7)$ \\
\hline $\mathrm{C} 2 \mathrm{E}-\mathrm{H} 2 \mathrm{EA}$ & 0.9900 \\
\hline $\mathrm{C} 2 \mathrm{E}-\mathrm{H} 2 \mathrm{~EB}$ & 0.9900 \\
\hline $\mathrm{C} 2 \mathrm{E}-\mathrm{C} 3 \mathrm{E}$ & $1.500(9)$ \\
\hline C3E-H3EA & 0.9900 \\
\hline C3E-H3EB & 0.9900 \\
\hline $\mathrm{C} 3 \mathrm{E}-\mathrm{N} 4 \mathrm{E}$ & $1.504(7)$ \\
\hline N4E-H4EA & 0.9100 \\
\hline N4E-H4EB & 0.9100 \\
\hline N4E-C5E & $1.480(7)$ \\
\hline C5E-H5EA & 0.9900 \\
\hline $\mathrm{C} 5 \mathrm{E}-\mathrm{H} 5 \mathrm{~EB}$ & 0.9900 \\
\hline C5E-C6E & $1.493(10)$ \\
\hline C6E-H6EA & 0.9900 \\
\hline C6E-H6EB & 0.9900 \\
\hline $\mathrm{O} 11 \mathrm{E}-\mathrm{C} 21 \mathrm{E}$ & $1.47(4)$ \\
\hline O11E-C61E & $1.41(5)$ \\
\hline $\mathrm{C} 21 \mathrm{E}-\mathrm{H} 21 \mathrm{C}$ & 0.9900 \\
\hline $\mathrm{C} 21 \mathrm{E}-\mathrm{H} 21 \mathrm{D}$ & 0.9900 \\
\hline $\mathrm{C} 21 \mathrm{E}-\mathrm{C} 31 \mathrm{E}$ & $1.55(5)$ \\
\hline $\mathrm{C} 31 \mathrm{E}-\mathrm{H} 31 \mathrm{C}$ & 0.9900 \\
\hline C31E-H31D & 0.9900 \\
\hline $\mathrm{C} 31 \mathrm{E}-\mathrm{N} 41 \mathrm{E}$ & $1.48(4)$ \\
\hline $\mathrm{N} 41 \mathrm{E}-\mathrm{H} 41 \mathrm{C}$ & 0.9100 \\
\hline N41E-H41D & 0.9100 \\
\hline $\mathrm{N} 41 \mathrm{E}-\mathrm{C} 51 \mathrm{E}$ & $1.55(4)$ \\
\hline C51E-H51C & 0.9900 \\
\hline C51E-H51D & 0.9900 \\
\hline $\mathrm{C} 51 \mathrm{E}-\mathrm{C} 61 \mathrm{E}$ & $1.45(4)$ \\
\hline C61E-H61C & 0.9900 \\
\hline C61E-H61D & 0.9900 \\
\hline $\mathrm{O} 1 \mathrm{D}-\mathrm{C} 2 \mathrm{D}$ & $1.430(9)$ \\
\hline $\mathrm{O} 1 \mathrm{D}-\mathrm{C} 6 \mathrm{D}$ & $1.417(9)$ \\
\hline $\mathrm{C} 2 \mathrm{D}-\mathrm{H} 2 \mathrm{DA}$ & 0.9900 \\
\hline $\mathrm{C} 2 \mathrm{D}-\mathrm{H} 2 \mathrm{DB}$ & 0.9900 \\
\hline $\mathrm{C} 2 \mathrm{D}-\mathrm{C} 3 \mathrm{D}$ & $1.479(10)$ \\
\hline $\mathrm{C} 3 \mathrm{D}-\mathrm{H} 3 \mathrm{DA}$ & 0.9900 \\
\hline $\mathrm{C} 3 \mathrm{D}-\mathrm{H} 3 \mathrm{DB}$ & 0.9900 \\
\hline C3D-N4D & $1.508(10)$ \\
\hline $\mathrm{N} 4 \mathrm{D}-\mathrm{H} 4 \mathrm{DA}$ & 0.9100 \\
\hline N4D-H4DB & 0.9100 \\
\hline
\end{tabular}




\begin{tabular}{|c|c|c|c|}
\hline $\mathrm{C} 5 \mathrm{~A}-\mathrm{H} 5 \mathrm{AB}$ & 0.9900 & $\mathrm{~N} 4 \mathrm{D}-\mathrm{C} 5 \mathrm{D}$ & $1.480(10)$ \\
\hline $\mathrm{C} 5 \mathrm{~A}-\mathrm{C} 6 \mathrm{~A}$ & $1.510(6)$ & $\mathrm{C} 5 \mathrm{D}-\mathrm{H} 5 \mathrm{DA}$ & 0.9900 \\
\hline C6A-H6AA & 0.9900 & C5D-H5DB & 0.9900 \\
\hline C6A-H6AB & 0.9900 & $\mathrm{C} 5 \mathrm{D}-\mathrm{C} 6 \mathrm{D}$ & $1.496(11)$ \\
\hline $\mathrm{O} 1 \mathrm{~B}-\mathrm{C} 2 \mathrm{~B}$ & $1.413(5)$ & C6D-H6DA & 0.9900 \\
\hline $\mathrm{O} 1 \mathrm{~B}-\mathrm{C} 6 \mathrm{~B}$ & $1.432(5)$ & C6D-H6DB & 0.9900 \\
\hline $\mathrm{C} 2 \mathrm{~B}-\mathrm{H} 2 \mathrm{BA}$ & 0.9900 & $\mathrm{O} 11 \mathrm{D}-\mathrm{C} 21 \mathrm{D}$ & $1.37(2)$ \\
\hline $\mathrm{C} 2 \mathrm{~B}-\mathrm{H} 2 \mathrm{BB}$ & 0.9900 & O11D-C61D & $1.44(2)$ \\
\hline $\mathrm{C} 2 \mathrm{~B}-\mathrm{C} 3 \mathrm{~B}$ & $1.516(5)$ & $\mathrm{C} 21 \mathrm{D}-\mathrm{H} 21 \mathrm{E}$ & 0.9900 \\
\hline $\mathrm{C} 3 \mathrm{~B}-\mathrm{H} 3 \mathrm{BA}$ & 0.9900 & $\mathrm{C} 21 \mathrm{D}-\mathrm{H} 21 \mathrm{~F}$ & 0.9900 \\
\hline $\mathrm{C} 3 \mathrm{~B}-\mathrm{H} 3 \mathrm{BB}$ & 0.9900 & $\mathrm{C} 21 \mathrm{D}-\mathrm{C} 31 \mathrm{D}$ & $1.54(2)$ \\
\hline $\mathrm{C} 3 \mathrm{~B}-\mathrm{N} 4 \mathrm{~B}$ & $1.494(5)$ & C31D-H31E & 0.9900 \\
\hline $\mathrm{N} 4 \mathrm{~B}-\mathrm{H} 4 \mathrm{BA}$ & 0.9100 & $\mathrm{C} 31 \mathrm{D}-\mathrm{H} 31 \mathrm{~F}$ & 0.9900 \\
\hline $\mathrm{N} 4 \mathrm{~B}-\mathrm{H} 4 \mathrm{BB}$ & 0.9100 & $\mathrm{C} 31 \mathrm{D}-\mathrm{N} 41 \mathrm{D}$ & $1.44(2)$ \\
\hline $\mathrm{N} 4 \mathrm{~B}-\mathrm{C} 5 \mathrm{~B}$ & $1.491(5)$ & N41D-H41E & 0.9100 \\
\hline $\mathrm{C} 5 \mathrm{~B}-\mathrm{H} 5 \mathrm{BA}$ & 0.9900 & $\mathrm{~N} 41 \mathrm{D}-\mathrm{H} 41 \mathrm{~F}$ & 0.9100 \\
\hline $\mathrm{C} 5 \mathrm{~B}-\mathrm{H} 5 \mathrm{BB}$ & 0.9900 & $\mathrm{~N} 41 \mathrm{D}-\mathrm{C} 51 \mathrm{D}$ & $1.54(3)$ \\
\hline $\mathrm{C} 5 \mathrm{~B}-\mathrm{C} 6 \mathrm{~B}$ & $1.497(6)$ & C51D-H51E & 0.9900 \\
\hline $\mathrm{C} 6 \mathrm{~B}-\mathrm{H} 6 \mathrm{BA}$ & 0.9900 & C51D-H51F & 0.9900 \\
\hline $\mathrm{C} 6 \mathrm{~B}-\mathrm{H} 6 \mathrm{BB}$ & 0.9900 & C51D-C61D & $1.52(2)$ \\
\hline $\mathrm{O} 1 \mathrm{C}-\mathrm{C} 2 \mathrm{C}$ & $1.403(9)$ & C61D-H61E & 0.9900 \\
\hline $\mathrm{O} 1 \mathrm{C}-\mathrm{C} 6 \mathrm{C}$ & $1.395(8)$ & C61D-H61F & 0.9900 \\
\hline $\mathrm{C} 2 \mathrm{C}-\mathrm{H} 2 \mathrm{CA}$ & 0.9900 & $\mathrm{O} 2 \mathrm{~W}-\mathrm{H} 2 \mathrm{WA}$ & 0.8486 \\
\hline $\mathrm{C} 2 \mathrm{C}-\mathrm{H} 2 \mathrm{CB}$ & 0.9900 & $\mathrm{O} 2 \mathrm{~W}-\mathrm{H} 2 \mathrm{WB}$ & 0.8488 \\
\hline $\mathrm{C} 2 \mathrm{C}-\mathrm{C} 3 \mathrm{C}$ & $1.505(9)$ & $\mathrm{O} 3 \mathrm{~W}-\mathrm{H} 3 \mathrm{WA}$ & 0.8504 \\
\hline $\mathrm{C} 3 \mathrm{C}-\mathrm{H} 3 \mathrm{CA}$ & 0.9900 & $\mathrm{O} 3 \mathrm{~W}-\mathrm{H} 3 \mathrm{WB}$ & 0.8517 \\
\hline $\mathrm{C} 3 \mathrm{C}-\mathrm{H} 3 \mathrm{CB}$ & 0.9900 & $\mathrm{O} 4 \mathrm{~W}-\mathrm{H} 4 \mathrm{WA}$ & 0.8512 \\
\hline $\mathrm{C} 3 \mathrm{C}-\mathrm{N} 4 \mathrm{C}$ & $1.484(8)$ & $\mathrm{O} 4 \mathrm{~W}-\mathrm{H} 4 \mathrm{WB}$ & 0.8530 \\
\hline $\mathrm{O} 1-\mathrm{Mo} 1-\mathrm{O} 2 \mathrm{H}$ & $82.96(11)$ & $\mathrm{C} 5 \mathrm{~B}-\mathrm{N} 4 \mathrm{~B}-\mathrm{H} 4 \mathrm{BB}$ & 109.5 \\
\hline $\mathrm{O} 1-\mathrm{Mo1}-\mathrm{O} 6 \mathrm{H}$ & $71.17(11)$ & $\mathrm{N} 4 \mathrm{~B}-\mathrm{C} 5 \mathrm{~B}-\mathrm{H} 5 \mathrm{BA}$ & 109.9 \\
\hline O6-Mo1-O1 & $149.82(11)$ & $\mathrm{N} 4 \mathrm{~B}-\mathrm{C} 5 \mathrm{~B}-\mathrm{H} 5 \mathrm{BB}$ & 109.9 \\
\hline $\mathrm{O} 6-\mathrm{Mo} 1-\mathrm{O} 2 \mathrm{H}$ & $71.81(10)$ & $\mathrm{N} 4 \mathrm{~B}-\mathrm{C} 5 \mathrm{~B}-\mathrm{C} 6 \mathrm{~B}$ & $109.1(3)$ \\
\hline $\mathrm{O} 6-\mathrm{Mo1}-\mathrm{O} 6 \mathrm{H}$ & $84.95(10)$ & $\mathrm{H} 5 \mathrm{BA}-\mathrm{C} 5 \mathrm{~B}-\mathrm{H} 5 \mathrm{BB}$ & 108.3 \\
\hline $\mathrm{O} 7-\mathrm{Mo1}-\mathrm{O} 1$ & $94.58(13)$ & $\mathrm{C} 6 \mathrm{~B}-\mathrm{C} 5 \mathrm{~B}-\mathrm{H} 5 \mathrm{BA}$ & 109.9 \\
\hline O7-Mo1-O6 & $101.90(12)$ & $\mathrm{C} 6 \mathrm{~B}-\mathrm{C} 5 \mathrm{~B}-\mathrm{H} 5 \mathrm{BB}$ & 109.9 \\
\hline $\mathrm{O} 7-\mathrm{Mo} 1-\mathrm{O} 2 \mathrm{H}$ & $90.82(12)$ & $\mathrm{O} 1 \mathrm{~B}-\mathrm{C} 6 \mathrm{~B}-\mathrm{C} 5 \mathrm{~B}$ & $111.8(3)$ \\
\hline $\mathrm{O} 7-\mathrm{Mo1}-\mathrm{O} 6 \mathrm{H}$ & $158.02(11)$ & $\mathrm{O} 1 \mathrm{~B}-\mathrm{C} 6 \mathrm{~B}-\mathrm{H} 6 \mathrm{BA}$ & 109.3 \\
\hline $\mathrm{O} 8-\mathrm{Mo1}-\mathrm{O} 1$ & $102.23(13)$ & $\mathrm{O} 1 \mathrm{~B}-\mathrm{C} 6 \mathrm{~B}-\mathrm{H} 6 \mathrm{BB}$ & 109.3 \\
\hline $\mathrm{O} 8-\mathrm{Mo1}-\mathrm{O} 6$ & $97.36(13)$ & $\mathrm{C} 5 \mathrm{~B}-\mathrm{C} 6 \mathrm{~B}-\mathrm{H} 6 \mathrm{BA}$ & 109.3 \\
\hline O8-Mo1—O7 & $105.90(14)$ & $\mathrm{C} 5 \mathrm{~B}-\mathrm{C} 6 \mathrm{~B}-\mathrm{H} 6 \mathrm{BB}$ & 109.3 \\
\hline $\mathrm{O} 8-\mathrm{Mo} 1-\mathrm{O} 2 \mathrm{H}$ & $161.86(13)$ & $\mathrm{H} 6 \mathrm{BA}-\mathrm{C} 6 \mathrm{~B}-\mathrm{H} 6 \mathrm{BB}$ & 107.9 \\
\hline $\mathrm{O} 8-\mathrm{Mol}-\mathrm{O} 6 \mathrm{H}$ & $93.71(12)$ & $\mathrm{C} 6 \mathrm{C}-\mathrm{O} 1 \mathrm{C}-\mathrm{C} 2 \mathrm{C}$ & $109.8(5)$ \\
\hline $\mathrm{O} 6 \mathrm{H}-\mathrm{Mo} 1-\mathrm{O} 2 \mathrm{H}$ & $71.30(9)$ & $\mathrm{O} 1 \mathrm{C}-\mathrm{C} 2 \mathrm{C}-\mathrm{H} 2 \mathrm{CA}$ & 109.2 \\
\hline $\mathrm{O} 1-\mathrm{Mo} 2-\mathrm{O} 2$ & $149.50(12)$ & $\mathrm{O} 1 \mathrm{C}-\mathrm{C} 2 \mathrm{C}-\mathrm{H} 2 \mathrm{CB}$ & 109.2 \\
\hline $\mathrm{O} 1-\mathrm{Mo} 2-\mathrm{O} 1 \mathrm{H}$ & $85.23(11)$ & $\mathrm{O} 1 \mathrm{C}-\mathrm{C} 2 \mathrm{C}-\mathrm{C} 3 \mathrm{C}$ & $112.0(5)$ \\
\hline $\mathrm{O} 1-\mathrm{Mo} 2-\mathrm{O} 6 \mathrm{H}$ & $70.54(11)$ & $\mathrm{H} 2 \mathrm{CA}-\mathrm{C} 2 \mathrm{C}-\mathrm{H} 2 \mathrm{CB}$ & 107.9 \\
\hline $\mathrm{O} 2-\mathrm{Mo} 2-\mathrm{O} 1 \mathrm{H}$ & $71.10(11)$ & $\mathrm{C} 3 \mathrm{C}-\mathrm{C} 2 \mathrm{C}-\mathrm{H} 2 \mathrm{CA}$ & 109.2 \\
\hline
\end{tabular}




\begin{tabular}{|c|c|}
\hline $\mathrm{O} 2-\mathrm{Mo} 2-\mathrm{O} 6 \mathrm{H}$ & $83.48(11)$ \\
\hline $\mathrm{O} 9-\mathrm{Mo} 2-\mathrm{O} 1$ & $96.60(15)$ \\
\hline $\mathrm{O} 9-\mathrm{Mo} 2-\mathrm{O} 2$ & $102.52(15)$ \\
\hline $\mathrm{O} 9-\mathrm{Mo} 2-\mathrm{O} 10$ & $105.99(18)$ \\
\hline $\mathrm{O} 9-\mathrm{Mo} 2-\mathrm{O} 1 \mathrm{H}$ & $90.80(14)$ \\
\hline $\mathrm{O} 9-\mathrm{Mo} 2-\mathrm{O} 6 \mathrm{H}$ & $158.22(14)$ \\
\hline $\mathrm{O} 10-\mathrm{Mo} 2-\mathrm{O} 1$ & $101.56(14)$ \\
\hline $\mathrm{O} 10-\mathrm{Mo} 2-\mathrm{O} 2$ & $95.77(15)$ \\
\hline $\mathrm{O} 10-\mathrm{Mo} 2-\mathrm{O} 1 \mathrm{H}$ & $160.83(15)$ \\
\hline $\mathrm{O} 10-\mathrm{Mo} 2-\mathrm{O} 6 \mathrm{H}$ & $94.04(15)$ \\
\hline $\mathrm{O} 6 \mathrm{H}-\mathrm{Mo} 2-\mathrm{O} 1 \mathrm{H}$ & $71.13(9)$ \\
\hline $\mathrm{O} 2-\mathrm{Mo3}-\mathrm{O} 1 \mathrm{H}$ & $71.25(11)$ \\
\hline $\mathrm{O} 2-\mathrm{Mo} 3-\mathrm{O} 5 \mathrm{H}$ & $83.28(11)$ \\
\hline $\mathrm{O} 3-\mathrm{Mo} 3-\mathrm{O} 2$ & $150.44(11)$ \\
\hline $\mathrm{O} 3-\mathrm{Mo} 3-\mathrm{O} 1 \mathrm{H}$ & $85.43(11)$ \\
\hline $\mathrm{O} 3-\mathrm{Mo} 3-\mathrm{O} 5 \mathrm{H}$ & $71.97(10)$ \\
\hline $\mathrm{O} 11-\mathrm{Mo} 3-\mathrm{O} 2$ & $101.54(14)$ \\
\hline O11-Mo3-O3 & $96.56(14)$ \\
\hline $\mathrm{O} 11-\mathrm{Mo} 3-\mathrm{O} 1 \mathrm{H}$ & $90.81(13)$ \\
\hline $\mathrm{O} 11-\mathrm{Mo} 3-\mathrm{O} 5 \mathrm{H}$ & $159.23(13)$ \\
\hline $\mathrm{O} 12-\mathrm{Mo} 3-\mathrm{O} 2$ & $94.92(14)$ \\
\hline $\mathrm{O} 12-\mathrm{Mo3}-\mathrm{O} 3$ & $102.24(13)$ \\
\hline $\mathrm{O} 12-\mathrm{Mo} 3-\mathrm{O} 11$ & $106.25(15)$ \\
\hline $\mathrm{O} 12-\mathrm{Mo} 3-\mathrm{O} 1 \mathrm{H}$ & $160.13(12)$ \\
\hline $\mathrm{O} 12-\mathrm{Mo} 3-\mathrm{O} 5 \mathrm{H}$ & $93.31(13)$ \\
\hline $\mathrm{O} 5 \mathrm{H}-\mathrm{Mo} 3-\mathrm{O} 1 \mathrm{H}$ & $71.43(9)$ \\
\hline $\mathrm{O} 3-\mathrm{Mo} 4-\mathrm{O} 4$ & $149.43(11)$ \\
\hline $\mathrm{O} 3-\mathrm{Mo} 4-\mathrm{O} 3 \mathrm{H}$ & $83.18(10)$ \\
\hline $\mathrm{O} 3-\mathrm{Mo} 4-\mathrm{O} 5 \mathrm{H}$ & $71.25(10)$ \\
\hline $\mathrm{O} 4-\mathrm{Mo} 4-\mathrm{O} 3 \mathrm{H}$ & $70.39(10)$ \\
\hline $\mathrm{O} 4-\mathrm{Mo} 4-\mathrm{O} 5 \mathrm{H}$ & $85.41(11)$ \\
\hline $\mathrm{O} 13-\mathrm{Mo} 4-\mathrm{O} 3$ & $103.68(13)$ \\
\hline $\mathrm{O} 13-\mathrm{Mo} 4-\mathrm{O} 4$ & $96.24(13)$ \\
\hline $\mathrm{O} 13-\mathrm{Mo} 4-\mathrm{O} 3 \mathrm{H}$ & $158.31(12)$ \\
\hline $\mathrm{O} 13-\mathrm{Mo} 4-\mathrm{O} 5 \mathrm{H}$ & $91.61(13)$ \\
\hline $\mathrm{O} 14-\mathrm{Mo} 4-\mathrm{O} 3$ & $96.60(13)$ \\
\hline $\mathrm{O} 14-\mathrm{Mo} 4-\mathrm{O} 4$ & $100.88(13)$ \\
\hline $\mathrm{O} 14-\mathrm{Mo} 4-\mathrm{O} 13$ & $103.89(15)$ \\
\hline $\mathrm{O} 14-\mathrm{Mo} 4-\mathrm{O} 3 \mathrm{H}$ & $95.57(12)$ \\
\hline $\mathrm{O} 14-\mathrm{Mo} 4-\mathrm{O} 5 \mathrm{H}$ & $162.40(12)$ \\
\hline $\mathrm{O} 5 \mathrm{H}-\mathrm{Mo} 4-\mathrm{O} 3 \mathrm{H}$ & $70.87(9)$ \\
\hline $\mathrm{O} 4-\mathrm{Mo5}-\mathrm{O} 5$ & $147.96(11)$ \\
\hline $\mathrm{O} 4-\mathrm{Mo} 5-\mathrm{O} 3 \mathrm{H}$ & $71.61(10)$ \\
\hline $\mathrm{O} 4-\mathrm{Mo5}-\mathrm{O} 4 \mathrm{H}$ & $83.53(11)$ \\
\hline $\mathrm{O} 5-\mathrm{Mo} 5-\mathrm{O} 3 \mathrm{H}$ & $83.23(10)$ \\
\hline $\mathrm{O} 5-\mathrm{Mo5}-\mathrm{O} 4 \mathrm{H}$ & $69.51(10)$ \\
\hline O15-Mo5-O4 & $97.32(12)$ \\
\hline $\mathrm{O} 15-\mathrm{Mo5}-\mathrm{O} 5$ & $100.87(12)$ \\
\hline
\end{tabular}

\begin{tabular}{|c|c|}
\hline $\mathrm{C} 3 \mathrm{C}-\mathrm{C} 2 \mathrm{C}-\mathrm{H} 2 \mathrm{CB}$ & 109.2 \\
\hline $\mathrm{C} 2 \mathrm{C}-\mathrm{C} 3 \mathrm{C}-\mathrm{H} 3 \mathrm{CA}$ & 109.7 \\
\hline $\mathrm{C} 2 \mathrm{C}-\mathrm{C} 3 \mathrm{C}-\mathrm{H} 3 \mathrm{CB}$ & 109.7 \\
\hline $\mathrm{H} 3 \mathrm{CA}-\mathrm{C} 3 \mathrm{C}-\mathrm{H} 3 \mathrm{CB}$ & 108.2 \\
\hline $\mathrm{N} 4 \mathrm{C}-\mathrm{C} 3 \mathrm{C}-\mathrm{C} 2 \mathrm{C}$ & $109.7(5)$ \\
\hline $\mathrm{N} 4 \mathrm{C}-\mathrm{C} 3 \mathrm{C}-\mathrm{H} 3 \mathrm{CA}$ & 109.7 \\
\hline $\mathrm{N} 4 \mathrm{C}-\mathrm{C} 3 \mathrm{C}-\mathrm{H} 3 \mathrm{CB}$ & 109.7 \\
\hline $\mathrm{C} 3 \mathrm{C}-\mathrm{N} 4 \mathrm{C}-\mathrm{H} 4 \mathrm{CA}$ & 109.3 \\
\hline $\mathrm{C} 3 \mathrm{C}-\mathrm{N} 4 \mathrm{C}-\mathrm{H} 4 \mathrm{CB}$ & 109.3 \\
\hline $\mathrm{C} 3 \mathrm{C}-\mathrm{N} 4 \mathrm{C}-\mathrm{C} 5 \mathrm{C}$ & $111.5(5)$ \\
\hline $\mathrm{H} 4 \mathrm{CA}-\mathrm{N} 4 \mathrm{C}-\mathrm{H} 4 \mathrm{CB}$ & 108.0 \\
\hline $\mathrm{C} 5 \mathrm{C}-\mathrm{N} 4 \mathrm{C}-\mathrm{H} 4 \mathrm{CA}$ & 109.3 \\
\hline $\mathrm{C} 5 \mathrm{C}-\mathrm{N} 4 \mathrm{C}-\mathrm{H} 4 \mathrm{CB}$ & 109.3 \\
\hline $\mathrm{N} 4 \mathrm{C}-\mathrm{C} 5 \mathrm{C}-\mathrm{H} 5 \mathrm{CA}$ & 110.1 \\
\hline $\mathrm{N} 4 \mathrm{C}-\mathrm{C} 5 \mathrm{C}-\mathrm{H} 5 \mathrm{CB}$ & 110.1 \\
\hline $\mathrm{H} 5 \mathrm{CA}-\mathrm{C} 5 \mathrm{C}-\mathrm{H} 5 \mathrm{CB}$ & 108.4 \\
\hline $\mathrm{C} 6 \mathrm{C}-\mathrm{C} 5 \mathrm{C}-\mathrm{N} 4 \mathrm{C}$ & $108.1(5)$ \\
\hline $\mathrm{C} 6 \mathrm{C}-\mathrm{C} 5 \mathrm{C}-\mathrm{H} 5 \mathrm{CA}$ & 110.1 \\
\hline $\mathrm{C} 6 \mathrm{C}-\mathrm{C} 5 \mathrm{C}-\mathrm{H} 5 \mathrm{CB}$ & 110.1 \\
\hline $\mathrm{O} 1 \mathrm{C}-\mathrm{C} 6 \mathrm{C}-\mathrm{C} 5 \mathrm{C}$ & $111.3(6)$ \\
\hline $\mathrm{O} 1 \mathrm{C}-\mathrm{C} 6 \mathrm{C}-\mathrm{H} 6 \mathrm{CA}$ & 109.4 \\
\hline $\mathrm{O} 1 \mathrm{C}-\mathrm{C} 6 \mathrm{C}-\mathrm{H} 6 \mathrm{CB}$ & 109.4 \\
\hline $\mathrm{C} 5 \mathrm{C}-\mathrm{C} 6 \mathrm{C}-\mathrm{H} 6 \mathrm{CA}$ & 109.4 \\
\hline $\mathrm{C} 5 \mathrm{C}-\mathrm{C} 6 \mathrm{C}-\mathrm{H} 6 \mathrm{CB}$ & 109.4 \\
\hline $\mathrm{H} 6 \mathrm{CA}-\mathrm{C} 6 \mathrm{C}-\mathrm{H} 6 \mathrm{CB}$ & 108.0 \\
\hline $\mathrm{C} 61 \mathrm{C}-\mathrm{O} 11 \mathrm{C}-\mathrm{C} 21 \mathrm{C}$ & $108.7(15)$ \\
\hline $\mathrm{O} 11 \mathrm{C}-\mathrm{C} 21 \mathrm{C}-\mathrm{H} 21 \mathrm{~A}$ & 109.7 \\
\hline $\mathrm{O} 11 \mathrm{C}-\mathrm{C} 21 \mathrm{C}-\mathrm{H} 21 \mathrm{~B}$ & 109.7 \\
\hline $\mathrm{O} 11 \mathrm{C}-\mathrm{C} 21 \mathrm{C}-\mathrm{C} 31 \mathrm{C}$ & $109.8(16)$ \\
\hline $\mathrm{H} 21 \mathrm{~A}-\mathrm{C} 21 \mathrm{C}-\mathrm{H} 21 \mathrm{~B}$ & 108.2 \\
\hline $\mathrm{C} 31 \mathrm{C}-\mathrm{C} 21 \mathrm{C}-\mathrm{H} 21 \mathrm{~A}$ & 109.7 \\
\hline $\mathrm{C} 31 \mathrm{C}-\mathrm{C} 21 \mathrm{C}-\mathrm{H} 21 \mathrm{~B}$ & 109.7 \\
\hline $\mathrm{C} 21 \mathrm{C}-\mathrm{C} 31 \mathrm{C}-\mathrm{H} 31 \mathrm{~A}$ & 110.1 \\
\hline $\mathrm{C} 21 \mathrm{C}-\mathrm{C} 31 \mathrm{C}-\mathrm{H} 31 \mathrm{~B}$ & 110.1 \\
\hline $\mathrm{H} 31 \mathrm{~A}-\mathrm{C} 31 \mathrm{C}-\mathrm{H} 31 \mathrm{~B}$ & 108.4 \\
\hline $\mathrm{N} 41 \mathrm{C}-\mathrm{C} 31 \mathrm{C}-\mathrm{C} 21 \mathrm{C}$ & $108.1(15)$ \\
\hline $\mathrm{N} 41 \mathrm{C}-\mathrm{C} 31 \mathrm{C}-\mathrm{H} 31 \mathrm{~A}$ & 110.1 \\
\hline $\mathrm{N} 41 \mathrm{C}-\mathrm{C} 31 \mathrm{C}-\mathrm{H} 31 \mathrm{~B}$ & 110.1 \\
\hline $\mathrm{C} 31 \mathrm{C}-\mathrm{N} 41 \mathrm{C}-\mathrm{H} 41 \mathrm{~A}$ & 109.2 \\
\hline $\mathrm{C} 31 \mathrm{C}-\mathrm{N} 41 \mathrm{C}-\mathrm{H} 41 \mathrm{~B}$ & 109.2 \\
\hline $\mathrm{C} 31 \mathrm{C}-\mathrm{N} 41 \mathrm{C}-\mathrm{C} 51 \mathrm{C}$ & $112.1(16)$ \\
\hline $\mathrm{H} 41 \mathrm{~A}-\mathrm{N} 41 \mathrm{C}-\mathrm{H} 41 \mathrm{~B}$ & 107.9 \\
\hline $\mathrm{C} 51 \mathrm{C}-\mathrm{N} 41 \mathrm{C}-\mathrm{H} 41 \mathrm{~A}$ & 109.2 \\
\hline $\mathrm{C} 51 \mathrm{C}-\mathrm{N} 41 \mathrm{C}-\mathrm{H} 41 \mathrm{~B}$ & 109.2 \\
\hline $\mathrm{N} 41 \mathrm{C}-\mathrm{C} 51 \mathrm{C}-\mathrm{H} 51 \mathrm{~A}$ & 109.7 \\
\hline $\mathrm{N} 41 \mathrm{C}-\mathrm{C} 51 \mathrm{C}-\mathrm{H} 51 \mathrm{~B}$ & 109.8 \\
\hline $\mathrm{N} 41 \mathrm{C}-\mathrm{C} 51 \mathrm{C}-\mathrm{C} 61 \mathrm{C}$ & $109.6(15)$ \\
\hline $\mathrm{H} 51 \mathrm{~A}-\mathrm{C} 51 \mathrm{C}-\mathrm{H} 51 \mathrm{~B}$ & 108.2 \\
\hline
\end{tabular}




$\begin{array}{ll}\text { O15-Mo5-O3H } & 161.18(11) \\ \text { O15-Mo5-O4H } & 92.81(12) \\ \text { O16-Mo5-O4 } & 103.40(13) \\ \text { O16-Mo5-O5 } & 96.86(12) \\ \text { O16-Mo5-O15 } & 105.52(14) \\ \text { O16-Mo5-O3H } & 92.07(12) \\ \text { O16-Mo5-O4H } & 159.17(12) \\ \text { O3H-Mo5-O4H } & 71.24(9) \\ \text { O5-Mo6-O2H } & 83.57(10) \\ \text { O5-Mo6-O4H } & 71.69(10) \\ \text { O6-Mo6-O5 } & 149.28(10) \\ \text { O6-Mo6-O2H } & 72.51(10) \\ \text { O6-Mo6-O4H } & 82.58(11) \\ \text { O17-Mo6-O5 } & 94.63(12) \\ \text { O17-Mo6-O6 } & 103.69(12) \\ \text { O17-Mo6-O2H } & 88.87(11) \\ \text { O17-Mo6-O4H } & 157.37(11) \\ \text { O18-Mo6-O5 } & 101.37(12) \\ \text { O18-Mo6-O6 } & 97.17(12) \\ \text { O18-Mo6-O17 } & 105.74(13) \\ \text { O18-Mo6-O2H } & 163.99(11) \\ \text { O18-Mo6-O4H } & 94.82(12) \\ \text { O4H-Mo6-O2H } & 72.08(9) \\ \text { Mo2-O1-Mo1 } & 119.53(15) \\ \text { Mo2-O2-Mo3 } & 120.08(15) \\ \text { Mo3-O3-Mo4 } & 118.91(13) \\ \text { Mo5-O4-Mo4 } & 120.31(14) \\ \text { Mo6-O5-Mo5 } & 119.87(13) \\ \text { Mo6-O6-Mo1 } & 118.99(13) \\ \text { H1WA-O1W-H1WB } & 140.1 \\ \text { O1H-Fe1-O2H } & 96.79(11) \\ \text { O1H-Fe1-O3H } & 97.53(11) \\ \text { O1H-Fe1-O4H } & 177.91(10) \\ \text { O1H-Fe1-O5H } & 84.54(11) \\ \text { O1H-Fe1-O6H } & 84.69(11) \\ \text { O2H-Fe1-O4H } & 83.25(10) \\ \text { O2H-Fe1-O6H } & 83.30(11) \\ \text { O3H-Fe1-O2H } & 99.02(11) \\ \text { O3H-Fe1-O4H } & 84.52(11) \\ \text { O3H-Fe1-O5H } & 84.35(11) \\ \text { O3H-Fe1-O6H } & 176.55(10) \\ \text { O5H-Fe1-O2H } & 176.15(10) \\ \text { O5H-Fe1-O4H } & 95.29(11) \\ \text { O5H-Fe1-O6H } & 93.25(11) \\ \text { O6H-Fe1-O4H } & 93.25(11) \\ \text { Mo2-O1H-H1H } & \\ \text { Mo3-O1H-Mo2 } & \\ \text { Mo3-O1H-H1H } & \\ & \end{array}$

$\begin{array}{ll}\text { C61C-C51C-H51A } & 109.7 \\ \text { C61C-C51C-H51B } & 109.8 \\ \text { O11C-C61C-C51C } & 111.3(16) \\ \text { O11C-C61C-H61A } & 109.4 \\ \text { O11C-C61C-H61B } & 109.4 \\ \text { C51C-C61C-H61A } & 109.4 \\ \text { C51C-C61C-H61B } & 109.4 \\ \text { H61A-C61C-H61B } & 108.0 \\ \text { C6E-O1E-C2E } & 109.8(5) \\ \text { O1E-C2E-H2EA } & 109.5 \\ \text { O1E-C2E-H2EB } & 109.5 \\ \text { O1E-C2E-C3E } & 110.5(5) \\ \text { H2EA-C2E-H2EB } & 108.1 \\ \text { C3E-C2E-H2EA } & 109.5 \\ \text { C3E-C2E-H2EB } & 109.5 \\ \text { C2E-C3E-H3EA } & 109.8 \\ \text { C2E-C3E-H3EB } & 109.8 \\ \text { C2E-C3E-N4E } & 109.5(5) \\ \text { H3EA-C3E-H3EB } & 108.2 \\ \text { N4E-C3E-H3EA } & 109.8 \\ \text { N4E-C3E-H3EB } & 109.8 \\ \text { C3E-N4E-H4EA } & 109.2 \\ \text { C3E-N4E-H4EB } & 109.2 \\ \text { H4EA-N4E-H4EB } & 107.9 \\ \text { C5E-N4E-C3E } & 111.9(4) \\ \text { C5E-N4E-H4EA } & 109.2 \\ \text { C5E-N4E-H4EB } & 109.2 \\ \text { N4E-C5E-H5EA } & 110.0 \\ \text { N4E-C5E-H5EB } & 110.0 \\ \text { N4E-C5E-C6E } & 108.7(5) \\ \text { H5EA-C5E-H5EB } & 108.3 \\ \text { C6E-C5E-H5EA } & 110.0 \\ \text { C6E-C5E-H5EB } & 110.0 \\ \text { O1E-C6E-C5E } & 110.9(5) \\ \text { O1E-C6E-H6EA } & 109.5 \\ \text { O1E-C6E-H6EB } & 109.5 \\ \text { C5E-C6E-H6EA } & 109.5 \\ \text { C5E-C6E-H6EB } & 109.5 \\ \text { H6EA-C6E-H6EB } & 108.0 \\ \text { C61E-O11E-C21E } & 108(3) \\ \text { O11E-C21E-H21C } & 109.6 \\ \text { O11E-C21E-H21D } & 109.6 \\ \text { O11E-C21E-C31E } & 110(3) \\ \text { H21C-C21E-H21D } & 108.1 \\ \text { C31E-C21E-H21C } & 109.6 \\ \text { C31E-C21E-H21D } & 109.6 \\ \text { C21E-C31E-H31C } & \\ \text { C21E-C31E-H31D } & \\ & \end{array}$




\begin{tabular}{|c|c|}
\hline $\mathrm{Fe} 1-\mathrm{O} 1 \mathrm{H}-\mathrm{Mo} 2$ & $102.45(11)$ \\
\hline $\mathrm{Fe} 1-\mathrm{O} 1 \mathrm{H}-\mathrm{Mo} 3$ & $101.86(11)$ \\
\hline $\mathrm{Fe} 1-\mathrm{O} 1 \mathrm{H}-\mathrm{H} 1 \mathrm{H}$ & 118.2 \\
\hline $\mathrm{Mo} 1-\mathrm{O} 2 \mathrm{H}-\mathrm{H} 2 \mathrm{H}$ & 118.5 \\
\hline Mo6-O2H-Mo1 & $92.61(9)$ \\
\hline $\mathrm{Mo6}-\mathrm{O} 2 \mathrm{H}-\mathrm{H} 2 \mathrm{H}$ & 118.5 \\
\hline $\mathrm{Fe} 1-\mathrm{O} 2 \mathrm{H}-\mathrm{Mo} 1$ & $102.24(10)$ \\
\hline $\mathrm{Fe} 1-\mathrm{O} 2 \mathrm{H}-\mathrm{Mo} 6$ & $102.24(11)$ \\
\hline $\mathrm{Fe} 1-\mathrm{O} 2 \mathrm{H}-\mathrm{H} 2 \mathrm{H}$ & 118.5 \\
\hline $\mathrm{Mo} 4-\mathrm{O} 3 \mathrm{H}-\mathrm{H} 3 \mathrm{H}$ & 117.7 \\
\hline $\mathrm{Mo5}-\mathrm{O} 3 \mathrm{H}-\mathrm{Mo} 4$ & $93.52(10)$ \\
\hline $\mathrm{Mo5}-\mathrm{O} 3 \mathrm{H}-\mathrm{H} 3 \mathrm{H}$ & 117.7 \\
\hline $\mathrm{Fe} 1-\mathrm{O} 3 \mathrm{H}-\mathrm{Mo} 4$ & $102.43(10)$ \\
\hline $\mathrm{Fe} 1-\mathrm{O} 3 \mathrm{H}-\mathrm{Mo5}$ & $103.95(11)$ \\
\hline $\mathrm{Fe} 1-\mathrm{O} 3 \mathrm{H}-\mathrm{H} 3 \mathrm{H}$ & 117.7 \\
\hline $\mathrm{Mo5}-\mathrm{O} 4 \mathrm{H}-\mathrm{H} 4 \mathrm{H}$ & 118.6 \\
\hline Mo6-O4H-Mo5 & $94.02(9)$ \\
\hline Mo6- $\mathrm{O} 4 \mathrm{H}-\mathrm{H} 4 \mathrm{H}$ & 118.6 \\
\hline $\mathrm{Fe} 1-\mathrm{O} 4 \mathrm{H}-\mathrm{Mo} 5$ & $100.27(10)$ \\
\hline $\mathrm{Fe} 1-\mathrm{O} 4 \mathrm{H}-\mathrm{Mo6}$ & $102.34(11)$ \\
\hline $\mathrm{Fe} 1-\mathrm{O} 4 \mathrm{H}-\mathrm{H} 4 \mathrm{H}$ & 118.6 \\
\hline $\mathrm{Mo} 3-\mathrm{O} 5 \mathrm{H}-\mathrm{Mo} 4$ & $93.37(9)$ \\
\hline $\mathrm{Mo} 3-\mathrm{O} 5 \mathrm{H}-\mathrm{H} 5 \mathrm{H}$ & 118.3 \\
\hline $\mathrm{Mo} 4-\mathrm{O} 5 \mathrm{H}-\mathrm{H} 5 \mathrm{H}$ & 118.3 \\
\hline $\mathrm{Fe} 1-\mathrm{O} 5 \mathrm{H}-\mathrm{Mo} 3$ & $102.11(11)$ \\
\hline $\mathrm{Fe} 1-\mathrm{O} 5 \mathrm{H}-\mathrm{Mo} 4$ & $102.35(11)$ \\
\hline $\mathrm{Fe} 1-\mathrm{O} 5 \mathrm{H}-\mathrm{H} 5 \mathrm{H}$ & 118.3 \\
\hline Mo1-O6H-Mo2 & $94.54(10)$ \\
\hline $\mathrm{Mo1}-\mathrm{O} 6 \mathrm{H}-\mathrm{H} 6 \mathrm{H}$ & 117.9 \\
\hline $\mathrm{Mo} 2-\mathrm{O} 6 \mathrm{H}-\mathrm{H} 6 \mathrm{H}$ & 117.9 \\
\hline $\mathrm{Fe} 1-\mathrm{O} 6 \mathrm{H}-\mathrm{Mo} 1$ & $103.15(11)$ \\
\hline $\mathrm{Fe} 1-\mathrm{O} 6 \mathrm{H}-\mathrm{Mo} 2$ & $101.72(11)$ \\
\hline $\mathrm{Fe} 1-\mathrm{O} 6 \mathrm{H}-\mathrm{H} 6 \mathrm{H}$ & 117.9 \\
\hline $\mathrm{O} 2 \mathrm{~S}-\mathrm{S} 1-\mathrm{O} 1 \mathrm{~S}$ & $108.74(16)$ \\
\hline $\mathrm{O} 3 \mathrm{~S}-\mathrm{S} 1-\mathrm{O} 1 \mathrm{~S}$ & $109.32(19)$ \\
\hline $\mathrm{O} 3 \mathrm{~S}-\mathrm{S} 1-\mathrm{O} 2 \mathrm{~S}$ & $109.95(19)$ \\
\hline $\mathrm{O} 3 \mathrm{~S}-\mathrm{S} 1-\mathrm{O} 4 \mathrm{~S}$ & $111.28(18)$ \\
\hline $\mathrm{O} 4 \mathrm{~S}-\mathrm{S} 1-\mathrm{O} 1 \mathrm{~S}$ & $108.9(2)$ \\
\hline $\mathrm{O} 4 \mathrm{~S}-\mathrm{S} 1-\mathrm{O} 2 \mathrm{~S}$ & $108.6(2)$ \\
\hline $\mathrm{C} 6 \mathrm{~A}-\mathrm{O} 1 \mathrm{~A}-\mathrm{C} 2 \mathrm{~A}$ & $109.2(3)$ \\
\hline $\mathrm{O} 1 \mathrm{~A}-\mathrm{C} 2 \mathrm{~A}-\mathrm{H} 2 \mathrm{AA}$ & 109.4 \\
\hline $\mathrm{O} 1 \mathrm{~A}-\mathrm{C} 2 \mathrm{~A}-\mathrm{H} 2 \mathrm{AB}$ & 109.4 \\
\hline $\mathrm{O} 1 \mathrm{~A}-\mathrm{C} 2 \mathrm{~A}-\mathrm{C} 3 \mathrm{~A}$ & $111.3(4)$ \\
\hline $\mathrm{H} 2 \mathrm{AA}-\mathrm{C} 2 \mathrm{~A}-\mathrm{H} 2 \mathrm{AB}$ & 108.0 \\
\hline $\mathrm{C} 3 \mathrm{~A}-\mathrm{C} 2 \mathrm{~A}-\mathrm{H} 2 \mathrm{AA}$ & 109.4 \\
\hline $\mathrm{C} 3 \mathrm{~A}-\mathrm{C} 2 \mathrm{~A}-\mathrm{H} 2 \mathrm{AB}$ & 109.4 \\
\hline $\mathrm{C} 2 \mathrm{~A}-\mathrm{C} 3 \mathrm{~A}-\mathrm{H} 3 \mathrm{AA}$ & 109.9 \\
\hline $\mathrm{C} 2 \mathrm{~A}-\mathrm{C}_{3} \mathrm{~A}-\mathrm{H} 3 \mathrm{AB}$ & \\
\hline
\end{tabular}

$\begin{array}{ll}\text { H31C-C31E-H31D } & 108.5 \\ \text { N41E-C31E-C21E } & 107(3) \\ \text { N41E-C31E-H31C } & 110.2 \\ \text { N41E-C31E-H31D } & 110.2 \\ \text { C31E-N41E-H41C } & 109.6 \\ \text { C31E-N41E-H41D } & 109.6 \\ \text { C31E-N41E-C51E } & 110(2) \\ \text { H41C-N41E-H41D } & 108.1 \\ \text { C51E-N41E-H41C } & 109.6 \\ \text { C51E-N41E-H41D } & 109.6 \\ \text { N41E-C51E-H51C } & 109.7 \\ \text { N41E-C51E-H51D } & 109.7 \\ \text { H51C-C51E-H51D } & 108.2 \\ \text { C61E-C51E-N41E } & 110(2) \\ \text { C61E-C51E-H51C } & 109.7\end{array}$

C61E-C51E-H51C $\quad 109.7$

C61E-C51E-H51D $\quad 109.7$

O11E-C61E-C51E 114 (3)

O11E-C61E-H61C 108.7

O11E-C61E-H61D $\quad 108.7$

C51E-C61E-H61C $\quad 108.7$

C51E-C61E-H61D $\quad 108.7$

H61C-C61E-H61D 107.6

C6D-O1D-C2D 109.7 (6)

O1D-C2D-H2DA 109.4

O1D-C2D-H2DB $\quad 109.4$

$\mathrm{O} 1 \mathrm{D}-\mathrm{C} 2 \mathrm{D}-\mathrm{C} 3 \mathrm{D} \quad 111.3(6)$

H2DA-C2D-H2DB $\quad 108.0$

C3D-C2D-H2DA $\quad 109.4$

$\mathrm{C} 3 \mathrm{D}-\mathrm{C} 2 \mathrm{D}-\mathrm{H} 2 \mathrm{DB} \quad 109.4$

$\mathrm{C} 2 \mathrm{D}-\mathrm{C} 3 \mathrm{D}-\mathrm{H} 3 \mathrm{DA} \quad 109.5$

C2D-C3D-H3DB $\quad 109.5$

$\mathrm{C} 2 \mathrm{D}-\mathrm{C} 3 \mathrm{D}-\mathrm{N} 4 \mathrm{D} \quad 110.8(6)$

H3DA-C3D-H3DB $\quad 108.1$

N4D - C3D-H3DA $\quad 109.5$

$\mathrm{N} 4 \mathrm{D}-\mathrm{C} 3 \mathrm{D}-\mathrm{H} 3 \mathrm{DB} \quad 109.5$

C3D-N4D-H4DA 109.3

C3D-N4D-H4DB $\quad 109.3$

H4DA-N4D-H4DB $\quad 108.0$

$\mathrm{C} 5 \mathrm{D}-\mathrm{N} 4 \mathrm{D}-\mathrm{C} 3 \mathrm{D} \quad 111.6(6)$

C5D-N4D-H4DA 109.3

C5D-N4D-H4DB $\quad 109.3$

N4D-C5D-H5DA $\quad 109.5$

N4D-C5D-H5DB $\quad 109.5$

N4D - C5D - C6D 110.8 (7)

H5DA-C5D-H5DB $\quad 108.1$

C6D-C5D-H5DA $\quad 109.5$

C6D-C5D-H5DB $\quad 109.5$

O1D-C6D-C5D $111.9(6)$ 


\begin{tabular}{|c|c|}
\hline $\mathrm{C} 2 \mathrm{~A}-\mathrm{C} 3 \mathrm{~A}-\mathrm{N} 4 \mathrm{~A}$ & $109.1(3)$ \\
\hline $\mathrm{H} 3 \mathrm{AA}-\mathrm{C} 3 \mathrm{~A}-\mathrm{H} 3 \mathrm{AB}$ & 108.3 \\
\hline $\mathrm{N} 4 \mathrm{~A}-\mathrm{C} 3 \mathrm{~A}-\mathrm{H} 3 \mathrm{AA}$ & 109.9 \\
\hline $\mathrm{N} 4 \mathrm{~A}-\mathrm{C} 3 \mathrm{~A}-\mathrm{H} 3 \mathrm{AB}$ & 109.9 \\
\hline $\mathrm{C} 3 \mathrm{~A}-\mathrm{N} 4 \mathrm{~A}-\mathrm{H} 4 \mathrm{AA}$ & 109.2 \\
\hline $\mathrm{C} 3 \mathrm{~A}-\mathrm{N} 4 \mathrm{~A}-\mathrm{H} 4 \mathrm{AB}$ & 109.2 \\
\hline $\mathrm{C} 3 \mathrm{~A}-\mathrm{N} 4 \mathrm{~A}-\mathrm{C} 5 \mathrm{~A}$ & $112.1(3)$ \\
\hline $\mathrm{H} 4 \mathrm{AA}-\mathrm{N} 4 \mathrm{~A}-\mathrm{H} 4 \mathrm{AB}$ & 107.9 \\
\hline $\mathrm{C} 5 \mathrm{~A}-\mathrm{N} 4 \mathrm{~A}-\mathrm{H} 4 \mathrm{AA}$ & 109.2 \\
\hline $\mathrm{C} 5 \mathrm{~A}-\mathrm{N} 4 \mathrm{~A}-\mathrm{H} 4 \mathrm{AB}$ & 109.2 \\
\hline $\mathrm{N} 4 \mathrm{~A}-\mathrm{C} 5 \mathrm{~A}-\mathrm{H} 5 \mathrm{AA}$ & 110.1 \\
\hline $\mathrm{N} 4 \mathrm{~A}-\mathrm{C} 5 \mathrm{~A}-\mathrm{H} 5 \mathrm{AB}$ & 110.1 \\
\hline $\mathrm{N} 4 \mathrm{~A}-\mathrm{C} 5 \mathrm{~A}-\mathrm{C} 6 \mathrm{~A}$ & $108.1(3)$ \\
\hline $\mathrm{H} 5 \mathrm{AA}-\mathrm{C} 5 \mathrm{~A}-\mathrm{H} 5 \mathrm{AB}$ & 108.4 \\
\hline $\mathrm{C} 6 \mathrm{~A}-\mathrm{C} 5 \mathrm{~A}-\mathrm{H} 5 \mathrm{AA}$ & 110.1 \\
\hline $\mathrm{C} 6 \mathrm{~A}-\mathrm{C} 5 \mathrm{~A}-\mathrm{H} 5 \mathrm{AB}$ & 110.1 \\
\hline $\mathrm{O} 1 \mathrm{~A}-\mathrm{C} 6 \mathrm{~A}-\mathrm{C} 5 \mathrm{~A}$ & $111.0(3)$ \\
\hline $\mathrm{O} 1 \mathrm{~A}-\mathrm{C} 6 \mathrm{~A}-\mathrm{H} 6 \mathrm{AA}$ & 109.4 \\
\hline $\mathrm{O} 1 \mathrm{~A}-\mathrm{C} 6 \mathrm{~A}-\mathrm{H} 6 \mathrm{AB}$ & 109.4 \\
\hline $\mathrm{C} 5 \mathrm{~A}-\mathrm{C} 6 \mathrm{~A}-\mathrm{H} 6 \mathrm{AA}$ & 109.4 \\
\hline $\mathrm{C} 5 \mathrm{~A}-\mathrm{C} 6 \mathrm{~A}-\mathrm{H} 6 \mathrm{AB}$ & 109.4 \\
\hline H6AA-C6A-H6AB & 108.0 \\
\hline $\mathrm{C} 2 \mathrm{~B}-\mathrm{O} 1 \mathrm{~B}-\mathrm{C} 6 \mathrm{~B}$ & $109.5(3)$ \\
\hline $\mathrm{O} 1 \mathrm{~B}-\mathrm{C} 2 \mathrm{~B}-\mathrm{H} 2 \mathrm{BA}$ & 109.4 \\
\hline $\mathrm{O} 1 \mathrm{~B}-\mathrm{C} 2 \mathrm{~B}-\mathrm{H} 2 \mathrm{BB}$ & 109.4 \\
\hline $\mathrm{O} 1 \mathrm{~B}-\mathrm{C} 2 \mathrm{~B}-\mathrm{C} 3 \mathrm{~B}$ & $111.1(3)$ \\
\hline $\mathrm{H} 2 \mathrm{BA}-\mathrm{C} 2 \mathrm{~B}-\mathrm{H} 2 \mathrm{BB}$ & 108.0 \\
\hline $\mathrm{C} 3 \mathrm{~B}-\mathrm{C} 2 \mathrm{~B}-\mathrm{H} 2 \mathrm{BA}$ & 109.4 \\
\hline $\mathrm{C} 3 \mathrm{~B}-\mathrm{C} 2 \mathrm{~B}-\mathrm{H} 2 \mathrm{BB}$ & 109.4 \\
\hline $\mathrm{C} 2 \mathrm{~B}-\mathrm{C} 3 \mathrm{~B}-\mathrm{H} 3 \mathrm{BA}$ & 110.0 \\
\hline $\mathrm{C} 2 \mathrm{~B}-\mathrm{C} 3 \mathrm{~B}-\mathrm{H} 3 \mathrm{BB}$ & 110.0 \\
\hline $\mathrm{H} 3 \mathrm{BA}-\mathrm{C} 3 \mathrm{~B}-\mathrm{H} 3 \mathrm{BB}$ & 108.3 \\
\hline $\mathrm{N} 4 \mathrm{~B}-\mathrm{C} 3 \mathrm{~B}-\mathrm{C} 2 \mathrm{~B}$ & $108.7(3)$ \\
\hline $\mathrm{N} 4 \mathrm{~B}-\mathrm{C} 3 \mathrm{~B}-\mathrm{H} 3 \mathrm{BA}$ & 110.0 \\
\hline $\mathrm{N} 4 \mathrm{~B}-\mathrm{C} 3 \mathrm{~B}-\mathrm{H} 3 \mathrm{BB}$ & 110.0 \\
\hline $\mathrm{C} 3 \mathrm{~B}-\mathrm{N} 4 \mathrm{~B}-\mathrm{H} 4 \mathrm{BA}$ & 109.5 \\
\hline $\mathrm{C} 3 \mathrm{~B}-\mathrm{N} 4 \mathrm{~B}-\mathrm{H} 4 \mathrm{BB}$ & 109.5 \\
\hline $\mathrm{H} 4 \mathrm{BA}-\mathrm{N} 4 \mathrm{~B}-\mathrm{H} 4 \mathrm{BB}$ & 108.0 \\
\hline $\mathrm{C} 5 \mathrm{~B}-\mathrm{N} 4 \mathrm{~B}-\mathrm{C} 3 \mathrm{~B}$ & $110.9(3)$ \\
\hline $\mathrm{C} 5 \mathrm{~B}-\mathrm{N} 4 \mathrm{~B}-\mathrm{H} 4 \mathrm{BA}$ & 109.5 \\
\hline $\mathrm{O} 1 \mathrm{~A}-\mathrm{C} 2 \mathrm{~A}-\mathrm{C} 3 \mathrm{~A}-\mathrm{N} 4 \mathrm{~A}$ & $56.9(4)$ \\
\hline $\mathrm{C} 2 \mathrm{~A}-\mathrm{O} 1 \mathrm{~A}-\mathrm{C} 6 \mathrm{~A}-\mathrm{C} 5 \mathrm{~A}$ & $64.4(4)$ \\
\hline $\mathrm{C} 2 \mathrm{~A}-\mathrm{C} 3 \mathrm{~A}-\mathrm{N} 4 \mathrm{~A}-\mathrm{C} 5 \mathrm{~A}$ & $-52.4(5)$ \\
\hline $\mathrm{C} 3 \mathrm{~A}-\mathrm{N} 4 \mathrm{~A}-\mathrm{C} 5 \mathrm{~A}-\mathrm{C} 6 \mathrm{~A}$ & $52.8(5)$ \\
\hline $\mathrm{N} 4 \mathrm{~A}-\mathrm{C} 5 \mathrm{~A}-\mathrm{C} 6 \mathrm{~A}-\mathrm{O} 1 \mathrm{~A}$ & $-58.7(4)$ \\
\hline $\mathrm{C} 6 \mathrm{~A}-\mathrm{O} 1 \mathrm{~A}-\mathrm{C} 2 \mathrm{~A}-\mathrm{C} 3 \mathrm{~A}$ & $-63.6(4)$ \\
\hline $\mathrm{O} 1 \mathrm{~B}-\mathrm{C} 2 \mathrm{~B}-\mathrm{C} 3 \mathrm{~B}-\mathrm{N} 4 \mathrm{~B}$ & $-58.7(4)$ \\
\hline
\end{tabular}

\begin{tabular}{|c|c|}
\hline $\mathrm{O} 1 \mathrm{D}-\mathrm{C} 6 \mathrm{D}-\mathrm{H} 6 \mathrm{DA}$ & 109.2 \\
\hline $\mathrm{O} 1 \mathrm{D}-\mathrm{C} 6 \mathrm{D}-\mathrm{H} 6 \mathrm{DB}$ & 109.2 \\
\hline $\mathrm{C} 5 \mathrm{D}-\mathrm{C} 6 \mathrm{D}-\mathrm{H} 6 \mathrm{DA}$ & 109.2 \\
\hline $\mathrm{C} 5 \mathrm{D}-\mathrm{C} 6 \mathrm{D}-\mathrm{H} 6 \mathrm{DB}$ & 109.2 \\
\hline $\mathrm{H} 6 \mathrm{DA}-\mathrm{C} 6 \mathrm{D}-\mathrm{H} 6 \mathrm{DB}$ & 107.9 \\
\hline $\mathrm{C} 21 \mathrm{D}-\mathrm{O} 11 \mathrm{D}-\mathrm{C} 61 \mathrm{D}$ & $111.7(13)$ \\
\hline $\mathrm{O} 11 \mathrm{D}-\mathrm{C} 21 \mathrm{D}-\mathrm{H} 21 \mathrm{E}$ & 109.6 \\
\hline $\mathrm{O} 11 \mathrm{D}-\mathrm{C} 21 \mathrm{D}-\mathrm{H} 21 \mathrm{~F}$ & 109.6 \\
\hline $\mathrm{O} 11 \mathrm{D}-\mathrm{C} 21 \mathrm{D}-\mathrm{C} 31 \mathrm{D}$ & $110.1(13)$ \\
\hline $\mathrm{H} 21 \mathrm{E}-\mathrm{C} 21 \mathrm{D}-\mathrm{H} 21 \mathrm{~F}$ & 108.2 \\
\hline $\mathrm{C} 31 \mathrm{D}-\mathrm{C} 21 \mathrm{D}-\mathrm{H} 21 \mathrm{E}$ & 109.6 \\
\hline $\mathrm{C} 31 \mathrm{D}-\mathrm{C} 21 \mathrm{D}-\mathrm{H} 21 \mathrm{~F}$ & 109.6 \\
\hline $\mathrm{C} 21 \mathrm{D}-\mathrm{C} 31 \mathrm{D}-\mathrm{H} 31 \mathrm{E}$ & 109.6 \\
\hline $\mathrm{C} 21 \mathrm{D}-\mathrm{C} 31 \mathrm{D}-\mathrm{H} 31 \mathrm{~F}$ & 109.6 \\
\hline $\mathrm{H} 31 \mathrm{E}-\mathrm{C} 31 \mathrm{D}-\mathrm{H} 31 \mathrm{~F}$ & 108.1 \\
\hline $\mathrm{N} 41 \mathrm{D}-\mathrm{C} 31 \mathrm{D}-\mathrm{C} 21 \mathrm{D}$ & $110.4(14)$ \\
\hline N41D $-\mathrm{C} 31 \mathrm{D}-\mathrm{H} 31 \mathrm{E}$ & 109.6 \\
\hline $\mathrm{N} 41 \mathrm{D}-\mathrm{C} 31 \mathrm{D}-\mathrm{H} 31 \mathrm{~F}$ & 109.6 \\
\hline $\mathrm{C} 31 \mathrm{D}-\mathrm{N} 41 \mathrm{D}-\mathrm{H} 41 \mathrm{E}$ & 110.0 \\
\hline $\mathrm{C} 31 \mathrm{D}-\mathrm{N} 41 \mathrm{D}-\mathrm{H} 41 \mathrm{~F}$ & 110.0 \\
\hline $\mathrm{C} 31 \mathrm{D}-\mathrm{N} 41 \mathrm{D}-\mathrm{C} 51 \mathrm{D}$ & $108.4(16)$ \\
\hline $\mathrm{H} 41 \mathrm{E}-\mathrm{N} 41 \mathrm{D}-\mathrm{H} 41 \mathrm{~F}$ & 108.4 \\
\hline $\mathrm{C} 51 \mathrm{D}-\mathrm{N} 41 \mathrm{D}-\mathrm{H} 41 \mathrm{E}$ & 110.0 \\
\hline $\mathrm{C} 51 \mathrm{D}-\mathrm{N} 41 \mathrm{D}-\mathrm{H} 41 \mathrm{~F}$ & 110.0 \\
\hline $\mathrm{N} 41 \mathrm{D}-\mathrm{C} 51 \mathrm{D}-\mathrm{H} 51 \mathrm{E}$ & 109.8 \\
\hline $\mathrm{N} 41 \mathrm{D}-\mathrm{C} 51 \mathrm{D}-\mathrm{H} 51 \mathrm{~F}$ & 109.8 \\
\hline $\mathrm{H} 51 \mathrm{E}-\mathrm{C} 51 \mathrm{D}-\mathrm{H} 51 \mathrm{~F}$ & 108.3 \\
\hline $\mathrm{C} 61 \mathrm{D}-\mathrm{C} 51 \mathrm{D}-\mathrm{N} 41 \mathrm{D}$ & $109.2(15)$ \\
\hline $\mathrm{C} 61 \mathrm{D}-\mathrm{C} 51 \mathrm{D}-\mathrm{H} 51 \mathrm{E}$ & 109.8 \\
\hline $\mathrm{C} 61 \mathrm{D}-\mathrm{C} 51 \mathrm{D}-\mathrm{H} 51 \mathrm{~F}$ & 109.8 \\
\hline $\mathrm{O} 11 \mathrm{D}-\mathrm{C} 61 \mathrm{D}-\mathrm{C} 51 \mathrm{D}$ & $108.5(13)$ \\
\hline $\mathrm{O} 11 \mathrm{D}-\mathrm{C} 61 \mathrm{D}-\mathrm{H} 61 \mathrm{E}$ & 110.0 \\
\hline $\mathrm{O} 11 \mathrm{D}-\mathrm{C} 61 \mathrm{D}-\mathrm{H} 61 \mathrm{~F}$ & 110.0 \\
\hline $\mathrm{C} 51 \mathrm{D}-\mathrm{C} 61 \mathrm{D}-\mathrm{H} 61 \mathrm{E}$ & 110.0 \\
\hline $\mathrm{C} 51 \mathrm{D}-\mathrm{C} 61 \mathrm{D}-\mathrm{H} 61 \mathrm{~F}$ & 110.0 \\
\hline $\mathrm{H} 61 \mathrm{E}-\mathrm{C} 61 \mathrm{D}-\mathrm{H} 61 \mathrm{~F}$ & 108.4 \\
\hline $\mathrm{H} 2 \mathrm{WA}-\mathrm{O} 2 \mathrm{~W}-\mathrm{H} 2 \mathrm{WB}$ & 109.7 \\
\hline $\mathrm{H} 3 \mathrm{WA}-\mathrm{O} 3 \mathrm{~W}-\mathrm{H} 3 \mathrm{WB}$ & 109.3 \\
\hline $\mathrm{H} 4 \mathrm{WA}-\mathrm{O} 4 \mathrm{~W}-\mathrm{H} 4 \mathrm{WB}$ & 109.1 \\
\hline $\mathrm{O} 1 \mathrm{E}-\mathrm{C} 2 \mathrm{E}-\mathrm{C} 3 \mathrm{E}-\mathrm{N} 4 \mathrm{E}$ & $55.3(7)$ \\
\hline $\mathrm{C} 2 \mathrm{E}-\mathrm{O} 1 \mathrm{E}-\mathrm{C} 6 \mathrm{E}-\mathrm{C} 5 \mathrm{E}$ & $64.1(7)$ \\
\hline $\mathrm{C} 2 \mathrm{E}-\mathrm{C} 3 \mathrm{E}-\mathrm{N} 4 \mathrm{E}-\mathrm{C} 5 \mathrm{E}$ & $-52.5(7)$ \\
\hline $\mathrm{C} 3 \mathrm{E}-\mathrm{N} 4 \mathrm{E}-\mathrm{C} 5 \mathrm{E}-\mathrm{C} 6 \mathrm{E}$ & $53.9(7)$ \\
\hline $\mathrm{N} 4 \mathrm{E}-\mathrm{C} 5 \mathrm{E}-\mathrm{C} 6 \mathrm{E}-\mathrm{O} 1 \mathrm{E}$ & $-59.7(7)$ \\
\hline $\mathrm{C} 6 \mathrm{E}-\mathrm{O} 1 \mathrm{E}-\mathrm{C} 2 \mathrm{E}-\mathrm{C} 3 \mathrm{E}$ & $-61.8(7)$ \\
\hline $\mathrm{O} 11 \mathrm{E}-\mathrm{C} 21 \mathrm{E}-\mathrm{C} 31 \mathrm{E}-\mathrm{N} 41 \mathrm{E}$ & $-61(4)$ \\
\hline
\end{tabular}




$\begin{array}{ll}\mathrm{C} 2 \mathrm{~B}-\mathrm{O} 1 \mathrm{~B}-\mathrm{C} 6 \mathrm{~B}-\mathrm{C} 5 \mathrm{~B} & -61.6(5) \\ \mathrm{C} 2 \mathrm{~B}-\mathrm{C} 3 \mathrm{~B}-\mathrm{N} 4 \mathrm{~B}-\mathrm{C} 5 \mathrm{~B} & 54.7(4) \\ \mathrm{C} 3 \mathrm{~B}-\mathrm{N} 4 \mathrm{~B}-\mathrm{C} 5 \mathrm{~B}-\mathrm{C} 6 \mathrm{~B} & -54.4(4) \\ \mathrm{N} 4 \mathrm{~B}-\mathrm{C} 5 \mathrm{~B}-\mathrm{C} 6 \mathrm{~B}-\mathrm{O} 1 \mathrm{~B} & 57.5(5) \\ \mathrm{C} 6 \mathrm{~B}-\mathrm{O} 1 \mathrm{~B}-\mathrm{C} 2 \mathrm{~B}-\mathrm{C} 3 \mathrm{~B} & 61.9(4) \\ \mathrm{O} 1 \mathrm{C}-\mathrm{C} 2 \mathrm{C}-\mathrm{C} 3 \mathrm{C}-\mathrm{N} 4 \mathrm{C} & -55.1(7) \\ \mathrm{C} 2 \mathrm{C}-\mathrm{O} 1 \mathrm{C}-\mathrm{C} 6 \mathrm{C}-\mathrm{C} 5 \mathrm{C} & -64.2(8) \\ \mathrm{C} 2 \mathrm{C}-\mathrm{C} 3 \mathrm{C}-\mathrm{N} 4 \mathrm{C}-\mathrm{C} 5 \mathrm{C} & 51.1(8) \\ \mathrm{C} 3 \mathrm{C}-\mathrm{N} 4 \mathrm{C}-\mathrm{C} 5 \mathrm{C}-\mathrm{C} 6 \mathrm{C} & -52.9(8) \\ \mathrm{N} 4 \mathrm{C}-\mathrm{C} 5 \mathrm{C}-\mathrm{C} 6 \mathrm{C}-\mathrm{O} 1 \mathrm{C} & 59.4(8) \\ \mathrm{C} 6 \mathrm{C}-\mathrm{O} 1 \mathrm{C}-\mathrm{C} 2 \mathrm{C}-\mathrm{C} 3 \mathrm{C} & 61.6(8) \\ \mathrm{O} 11 \mathrm{C}-\mathrm{C} 21 \mathrm{C}-\mathrm{C} 31 \mathrm{C}-\mathrm{N} 41 \mathrm{C} & 61(3) \\ \mathrm{C} 21 \mathrm{C}-\mathrm{O} 11 \mathrm{C}-\mathrm{C} 61 \mathrm{C}-\mathrm{C} 51 \mathrm{C} & 65(3) \\ \mathrm{C} 21 \mathrm{C}-\mathrm{C} 31 \mathrm{C}-\mathrm{N} 41 \mathrm{C}-\mathrm{C} 51 \mathrm{C} & -52(3) \\ \mathrm{C} 31 \mathrm{C}-\mathrm{N} 41 \mathrm{C}-\mathrm{C} 51 \mathrm{C}-\mathrm{C} 61 \mathrm{C} & 50(4) \\ \mathrm{N} 41 \mathrm{C}-\mathrm{C} 51 \mathrm{C}-\mathrm{C} 61 \mathrm{C}-\mathrm{O} 11 \mathrm{C} & -55(3) \\ \mathrm{C} 61 \mathrm{C}-\mathrm{O} 11 \mathrm{C}-\mathrm{C} 21 \mathrm{C}-\mathrm{C} 31 \mathrm{C} & -67(3) \\ \end{array}$

$\begin{array}{ll}\text { C21E-O11E-C61E-C51E } & -63(4) \\ \text { C21E-C31E-N41E-C51E } & 55(3) \\ \text { C31E-N41E-C51E-C61E } & -53(3) \\ \text { N41E-C51E-C61E-O11E } & 57(4) \\ \text { C61E-O11E-C21E-C31E } & 63(4) \\ \text { O1D-C2D-C3D-N4D } & -56.2(9) \\ \text { C2D-O1D-C6D-C5D } & -61.6(9) \\ \text { C2D-C3D-N4D-C5D } & 49.8(10) \\ \text { C3D-N4D-C5D-C6D } & -48.9(10) \\ \text { N4D-C5D-C6D-O1D } & 55.4(10) \\ \text { C6D-O1D-C2D-C3D } & 62.2(8) \\ \text { O11D-C21D-C31D-N41D } & 59.6(19) \\ \text { C21D-O11D-C61D-C51D } & 63.0(17) \\ \text { C21D-C31D-N41D-C51D } & -55.8(18) \\ \text { C31D-N41D-C51D-C61D } & 57.0(19) \\ \text { N41D-C51D-C61D-O11D } & -58.6(18) \\ \text { C61D-O11D-C21D-C31D } & -62.2(17)\end{array}$

Hydrogen-bond geometry $\left(\AA,{ }^{\circ}\right)$

\begin{tabular}{lllll}
\hline$D-\mathrm{H} \cdots A$ & $D-\mathrm{H}$ & $\mathrm{H} \cdots A$ & $D \cdots A$ & $D-\mathrm{H}^{\cdots} A$ \\
\hline $\mathrm{O} 1 W-\mathrm{H} 1 W A \cdots \mathrm{O} 9$ & 0.84 & 1.94 & $2.781(11)$ & 178 \\
$\mathrm{O} 1 W-\mathrm{H} 1 W B \cdots \mathrm{O} 10^{\mathrm{i}}$ & 0.85 & 1.99 & $2.831(11)$ & 173 \\
$\mathrm{O} 2 W-\mathrm{H} 2 W A \cdots \mathrm{O} 4 W^{\mathrm{ii}}$ & 0.85 & 1.80 & $2.619(4)$ & 161 \\
$\mathrm{O} 2 W-\mathrm{H} 2 W B \cdots \mathrm{O} 2 S^{\mathrm{ii}}$ & 0.85 & 2.05 & $2.876(4)$ & 164 \\
$\mathrm{O} 2 W-\mathrm{H} 2 W B \cdots \mathrm{O} 4 S^{\mathrm{ii}}$ & 0.85 & 2.48 & $3.129(5)$ & 133 \\
$\mathrm{O} 3 W-\mathrm{H} 3 W A \cdots \mathrm{O} 1 S^{\mathrm{ii}}$ & 0.85 & 1.99 & $2.790(5)$ & 155 \\
$\mathrm{O} 3 W-\mathrm{H} 3 W B \cdots \mathrm{O} 3$ & 0.85 & 1.92 & $2.753(4)$ & 167 \\
$\mathrm{O} 4 W-\mathrm{H} 4 W A \cdots \mathrm{O} 17^{\mathrm{iii}}$ & 0.85 & 1.88 & $2.714(4)$ & 165 \\
$\mathrm{O} 4 W-\mathrm{H} 4 W B \cdots \mathrm{O} 6$ & 0.85 & 1.94 & $2.761(4)$ & 161 \\
$\mathrm{O} 1 H-\mathrm{H} 1 H \cdots \mathrm{O} 4 S^{\mathrm{ii}}$ & 1.00 & 1.69 & $2.673(4)$ & 165 \\
$\mathrm{O} 2 H-\mathrm{H} 2 H \cdots \mathrm{O} 2 W$ & 1.00 & 1.78 & $2.743(4)$ & 162 \\
$\mathrm{O} 3 H-\mathrm{H} 3 H \cdots \mathrm{O} 3 S^{\mathrm{ii}}$ & 1.00 & 1.61 & $2.602(4)$ & 174 \\
$\mathrm{O} 4 H-\mathrm{H} 4 H \cdots \mathrm{O} 2 S$ & 1.00 & 2.13 & $2.911(4)$ & 133 \\
$\mathrm{O} 5 H-\mathrm{H} 5 H \cdots \mathrm{O} 1 S$ & 1.00 & 1.69 & $2.672(4)$ & 165 \\
$\mathrm{O} 6 H-\mathrm{H} 6 H \cdots \mathrm{O} 2 S$ & 1.00 & 1.83 & $2.691(4)$ & 142 \\
$\mathrm{~N} 4 A-\mathrm{H} 4 A A \cdots \mathrm{O} 4 W^{\mathrm{ii}}$ & 0.91 & 2.34 & $3.102(5)$ & 141 \\
$\mathrm{~N} 4 A-\mathrm{H} 4 A B \cdots \mathrm{O} 5$ & 0.91 & 1.86 & $2.760(4)$ & 169 \\
$\mathrm{~N} 4 B-\mathrm{H} 4 B A \cdots \mathrm{O} 4$ & 0.91 & 2.00 & $2.761(4)$ & 140 \\
$\mathrm{~N} 4 B-\mathrm{H} 4 B A \cdots \mathrm{O} 1 A^{\mathrm{ii}}$ & 0.91 & 2.26 & $2.840(4)$ & 121 \\
$\mathrm{~N} 4 B-\mathrm{H} 4 B B \cdots \mathrm{O} 15^{\mathrm{v}}$ & 0.91 & 1.97 & $2.869(4)$ & 171 \\
$\mathrm{~N} 4 C-\mathrm{H} 4 C A \cdots \mathrm{O} 1 E^{\mathrm{vi}}$ & 0.91 & 1.97 & $2.817(7)$ & 155 \\
$\mathrm{~N} 41 C-\mathrm{H} 41 A \cdots \mathrm{O} 11 E^{\mathrm{vi}}$ & 0.91 & 1.92 & $2.54(5)$ & 124 \\
$\mathrm{~N} 4 E-\mathrm{H} 4 E A \cdots \mathrm{O} 1$ & 0.91 & 1.91 & $2.780(6)$ & 160 \\
$\mathrm{~N} 41 E-\mathrm{H} 41 D \cdots \mathrm{O} 1$ & 0.91 & 1.52 & $2.35(3)$ & 150 \\
$\mathrm{~N} 4 D-\mathrm{H} 4 D A \cdots \mathrm{O} 1 S$ & 0.91 & 1.99 & $2.866(8)$ & 162 \\
& & &
\end{tabular}


supporting information

$\begin{array}{llllr}\mathrm{N} 4 D-\mathrm{H} 4 D B \cdots \mathrm{O} 2 & 0.91 & 2.00 & 2.846(9) & 155 \\ \mathrm{~N} 41 D-\mathrm{H} 41 E \cdots \mathrm{O} 2 & 0.91 & 1.62 & 2.520(19) & 169\end{array}$

Symmetry codes: (i) $-x,-y+2,-z+2$; (ii) $x+1, y, z$; (iii) $-x,-y+2,-z+1$; (iv) $-x+1,-y+1,-z+1$; (v) $-x,-y+1,-z+1$; (vi) $x-1, y-1, z$. 